ISSN 2075-4701

www.mdpi.com/journal/metals/

Review

\title{
Transformation Volume Effects on Shape Memory Alloys
}

\section{Volodymyr A. Chernenko ${ }^{1,2, *}$, Victor A. L'vov ${ }^{3,4}$, Eduard Cesari ${ }^{5}$, Anna Kosogor ${ }^{4}$ and Jose M. Barandiaran ${ }^{1}$}

1 BCMaterials, University of the Basque Country (UPV/EHU), Bilbao 48080, Spain;

E-Mail: manub@we.lc.ehu.es

2 Ikerbasque, Basque Foundation for Science, Bilbao 48011, Spain

3 Department of Radiophysics, Taras Shevchenko University, Glushkov Str. 2, build. 5, Kyiv 01601, Ukraine; E-Mail: victor.a.lvov@gmail.com

4 Institute of Magnetism, 36-b Vernadsky Blvd, Kyiv 03142, Ukraine; E-Mail: annakosogor@gmail.com

5 Department of Physics, University of Balearic Islands, Ctra. de Valldemossa, km 7.5, Palma de Mallorca 07122, Spain; E-Mail: eduard.cesari@uib.cat

* Author to whom correspondence should be addressed; E-Mail: vladimir.chernenko@gmail.com; Tel.: +34-946-015-130; Fax: +34-946-013-071.

Received: 1 April 2013; in revised form: 12 June 2013 / Accepted: 13 June 2013 /

Published: 2 July 2013

\begin{abstract}
It is generally accepted that the martensitic transformations (MTs) in the shape memory alloys (SMAs) are mainly characterized by the shear deformation of the crystal lattice that arises in the course of MT, while a comparatively small volume change during MT is considered as the secondary effect, which can be disregarded when the basic characteristics of MTs and functional properties of SMAs are analyzed. This point of view is a subject to change nowadays due to the new experimental and theoretical findings. The present article elucidates (i) the newly observed physical phenomena in different SMAs in their relation to the volume effect of MT; (ii) the theoretical analysis of the aforementioned volume-related phenomena.
\end{abstract}

Keywords: shape memory alloy; volume change; elastic modulus; crystal defects; martensite stabilization; entropy change 


\section{Introduction}

The widely used functional properties of the shape memory alloys (SMAs) stem from the martensitic transformation (MT) they exhibit during cooling or under the action of external fields of a different nature. The most important feature of martensitic transformation is the appearance of uniform shear strain of crystal lattice below the MT temperature resulting in the formation of martensitic phase [1-3]. Furthermore, MT is accompanied by the local shears within an invariant crystal lattice of the martensitic phase. These shears proceed in different directions and cause formation of the specific microstructure, which consists of the physically equivalent domains of the low temperature martensitic phase with the differently oriented crystallographic axes. Because of their physical equivalence, the domains of martensitic phase (martensite variants) are separated by the coherent, and therefore mobile, interfaces. The shear character of MT strains and appearance/presence of mobile interfaces give rise to a complicate thermoelastic behavior of the shape memory alloys and their engineering and medical applications [3-7]. The most of these applications are based on the shape memory effect and accessibility of large strain values under the moderate mechanical loading.

The ferromagnetic and metamagnetic shape memory alloys (FSMAs and MetaMSMAs) form a special class of the multifunctional materials (see [8-14] and references therein). These materials undergo both the ferromagnetic ordering and martensitic transformation (MT) on cooling. The considerable change in the martensitic structure of some FSMAs under the magnetic field occurs due to the extremely high mobility of coherent interfaces between the martensite variants. These changes cause the giant (up to $12 \%$ ) magnetically induced deformation under the moderate (about $0.1 \mathrm{~T}$ or even less) magnetic field [15-17].

The martensitic transformations are accompanied by the small volume changes. The value of volume change (that is the volume effect of MT) was recognized since early development of SMAs as an important factor influencing the thermoelastic character of MT [1,2,18]. Particularly, a reduced volume effect during MT was observed in Fe-Ni-Co-Ti alloys [19]; it appeared as one of the crucial factors ensuring thermoelastic character of MT in these unique alloys [20-23].

The early experiments with the SMAs under hydrostatic pressure $P$ have readily shown the interdependence between volume of SMA and characteristic MT temperature $T_{0}$ [24-27]. Depending on the sign of volume change during MT the hydrostatic pressure increases or decreases MT temperatures. The positive sign of $\mathrm{d} T_{0} / \mathrm{d} P$ is observed, e.g., for the Au-Cd, Ti-Ni and Ni-Mn-Ga alloys [24,25,28], while Fe-Ni-Co-Ti SMAs show a strong negative slope of MT temperature as a function of hydrostatic pressure [26,27].

Generally, the volume changes caused by MT, hydrostatic pressure or external magnetic field are considered mainly as a subject of academic interest. Nevertheless, these issues applied to SMAs have also practical importance as already mentioned above. No wonder, the relevant studies are still continuing (e.g., [29]). In particular, the intriguing results were obtained recently for the MTs in FSMAs and MetaMSMAs. First, the decisive role of volume magnetostriction in the kinetics of a coupled magnetostructural phase transformation of Ni-Mn-Ga alloy was demonstrated [30]. Second, the large contribution of the magnetic subsystem to the entropy change $\Delta S$ accompanying the MTs of the SMAs was discovered: it has been found that $\Delta S$ strongly decreases with the distance between the MT temperature and the Curie point $T_{C}$ [31-33]. As far as this behavior of the entropy change 
appeared to be inherent to the magnetic SMAs belonging to the different alloy systems, the spontaneous volume magnetostriction arising at the Curie point was assumed to be the universal factor that stipulates the magnetic contribution to the entropy change [33]. This assumption was confirmed by the quantitative theoretical analysis of the experimental data available for the Ni-Mn-Ga FSMAs. Third, the drastic influence of strong magnetic field $(1 \mathrm{~T}<H<20 \mathrm{~T})$ on the MTs of Ni-Mn-In and Ni-Mn-Sn MetaMSMAs was discovered (see [32,34] and references therein). In particular, the so-called "magnetic arrest" of forward MT and magnetically induced reverse MT were observed. Inasmuch as the driving force (the difference of the Gibbs potential of two phases) for the thermally induced MT is given by the product $\Delta S \Delta T$, where $\Delta T$ is the supercooling below the phase equilibrium temperature, the arrest of forward MT is promoted by the combination of two physical effects, which are the decrease of the forward MT temperature $T_{M F}$ measured for the Ni-Mn-X (X $\left.=\mathrm{In}, \mathrm{Sn}, \mathrm{Sb}\right)$ alloys under the elevated magnetic field and the decrease of $\Delta S$ value caused by the increase of the difference $T_{C}-T_{M F}$ [32]. A substantial contribution of spontaneous volume magnetostriction to the entropy change [33] suggests an idea about the role of forced volume magnetostriction in the arrest of forward MT and magnetic assistance to reverse MT.

Geometrically, the shear deformation of crystal lattice can be performed without the volume change. However, the rigorous theoretical analysis shows, that the shear deformation of crystal lattice and, in particular, the martensitic transformations are always accompanied by the volume change although the tendency to volume conservation exists in the literature and retards the measurement of volume change (see [28] and references therein). Furthermore, a consistent theoretical consideration shows that the crystal defects formation/redistribution during/after MT is also accompanied by the volume change. This volume change is thermodynamically conjugated to the "internal pressure" [35], which can stabilize [36] or destabilize [37] martensitic phase, depending on the type of MT and conditions of experiment.

Both stabilization and destabilization of martensitic state changes the functional properties of SMA, and therefore, the consideration of stabilizing and destabilizing pressures induced by the by external forces or internal factors is not only of academic interest but of practical importance as well.

In the present review article, a systematic analysis of physical effects related to the volume change during MT is presented. The analysis is based on the formal resemblance between the hydrostatic pressure produced by the hydrostatic mechanical load and internal pressure created by the crystal defects or thermodynamically conjugated to volume magnetostriction. This resemblance is substantiated in Section 2 using the symmetry conforming theory of cubic-tetragonal and cubic-rhombohedral MTs. The effect of hydrostatic pressure on the MT temperature, MT strain and soft elastic modulus of SMA is considered in Section 3. The martensite stabilization and destabilization caused by the martensite aging and cyclic MTs is considered in Sections 4 and 5, respectively. The role of the internal pressure in the appearance quasi-second-order MTs is discussed in Section 6. The experimental data that are relevant to the cubic-rhombohedral and cubic tetragonal MTs in the Ti-Ni, Au-Cd and Ni-Mn-Ga are used in the Sections 3-5 for the quantitative estimations. In Section 7, the magnetic contribution to the entropy change during the MT is related to the volume magnetostriction and evaluated for the Ni-Mn-Ga and Ni-Fe-Ga alloys. A reasonable agreement between the theoretical results and experimental data is stated. As a whole, this review uncovers the unprecedented role of 
volume variation due to MT, defects reconfiguration or magnetostriction in the phenomena exhibited by practically important martensitic multifunctional materials.

\section{Quantitative Theory of Thermoelastic Behavior of SMAs}

\subsection{Symmetry Conforming Approach to the Problem}

The SMAs undergo phase transformations from high-symmetry (austenitic) to low-symmetry (martensitic) phase. The tendency to the minimum of elastic energy is commonly considered as the basic principle of transformational behavior of SMAs. To use this principle for the description of functional properties of SMAs, the elastic energy is expanded as a series in the strain tensor components $\varepsilon_{i k}$. The mathematical structure of series is prescribed by the symmetry of crystal lattice: the series contains all combinations of strain tensor components, which are invariant with respect to the symmetry group of the crystal lattice in the high-symmetry (cubic) phase. The invariant series constructed for the different symmetry groups are presented in [38].

The elastic part of the Helmholtz free energy of cubic crystal can be expressed in terms of the linear combinations of strain tensor components, which are the basic functions of the one-dimensional, two-dimensional and three-dimensional irreducible representations of cubic group. The basic function of one-dimensional representation

$$
u_{1}=\left(\varepsilon_{x x}+\varepsilon_{y y}+\varepsilon_{z z}\right) / 3
$$

is invariant with respect to the cubic symmetry group, while the basic functions

$$
u_{2}=\sqrt{3}\left(\varepsilon_{x x}-\varepsilon_{y y}\right), u_{3}=2 \varepsilon_{z z}-\varepsilon_{y y}-\varepsilon_{x x},
$$

and

$$
u_{4}=\varepsilon_{y z}, \quad u_{5}=\varepsilon_{x z}, \quad u_{6}=\varepsilon_{x y},
$$

of the two-dimensional and three-dimensional representations are transformed with the help of $2 \times 2$ and $3 \times 3$ square matrices, respectively. (The coordinate axes are parallel to $<100>$ crystallographic directions of the cubic crystal). The free energy of the crystal undergoing MT is expressed as

$$
\begin{aligned}
F & =\frac{1}{2} c_{1} u_{1}^{2}+\frac{1}{2} c_{2}\left(u_{2}^{2}+u_{3}^{2}\right)+\frac{1}{2} c_{3}\left(u_{4}^{2}+u_{5}^{2}+u_{6}^{2}\right)+\frac{1}{3} a_{1} u_{1}^{3}+\frac{1}{2} a_{2} u_{1}\left(u_{2}^{2}+u_{3}^{2}\right) \\
& +\frac{1}{2} a_{3} u_{1}\left(u_{4}^{2}+u_{5}^{2}+u_{6}^{2}\right)+\frac{1}{3} a_{4} u_{3}\left(u_{3}^{2}-3 u_{2}^{2}\right)+a_{5} u_{4} u_{5} u_{6} \\
& +\frac{1}{2} a_{6}\left[u_{3}\left(2 u_{6}^{2}-u_{5}^{2}-u_{4}^{2}\right)+\sqrt{3} u_{2}\left(u_{5}^{2}-u_{4}^{2}\right)\right]+\frac{1}{4} b_{1} u_{1}^{4}+\frac{1}{2} b_{2} u_{1}^{2}\left(u_{2}^{2}+u_{3}^{2}\right) \\
& +\frac{1}{2} b_{3} u_{1}^{2}\left(u_{4}^{2}+u_{5}^{2}+u_{6}^{2}\right)+\frac{1}{4} b_{4}\left(u_{2}^{2}+u_{3}^{2}\right)^{2}+\frac{1}{4} b_{5}\left(u_{4}^{2}+u_{5}^{2}+u_{6}^{2}\right)^{2} \\
& +\frac{1}{2} b_{6}\left(u_{2}^{2}+u_{3}^{2}\right)\left(u_{4}^{2}+u_{5}^{2}+u_{6}^{2}\right)+\frac{1}{2} b_{7} u_{1} u_{3}\left(u_{3}^{2}-3 u_{2}^{2}\right)+b_{8} u_{1} u_{4} u_{5} u_{6} \\
& +\frac{1}{2} b_{9} u_{1}\left[u_{3}\left(2 u_{6}^{2}-u_{5}^{2}-u_{4}^{2}\right)+\sqrt{3} u_{2}\left(u_{5}^{2}-u_{4}^{2}\right)\right]+\frac{1}{4} b_{10}\left(u_{4}^{4}+u_{5}^{4}+u_{6}^{4}\right),
\end{aligned}
$$

where the coefficients

$$
c_{1}=3\left(C_{11}+2 C_{12}\right), c_{2}=\left(C_{11}-C_{12}\right) / 6 \equiv C^{\prime} / 3, c_{3}=4 C_{44}
$$


are expressed through the elastic modules $C_{\alpha, \beta}(\alpha, \beta=1,2, \ldots 6)$ of the crystal in the cubic phase, the coefficients of third-order and fourth-order energy terms are the linear combinations of the third-, and fourth-order elastic modules in the cubic phase [28,39].

The Gibbs potential of SMA is presented by the equation

$$
G=F-3 \sigma_{1} u_{1}-\frac{1}{6}\left(\sigma_{2} u_{2}+\sigma_{3} u_{3}\right)-2\left(\sigma_{4} u_{4}+\sigma_{5} u_{5}+\sigma_{6} u_{6}\right)
$$

where

$$
\begin{gathered}
\sigma_{1}=-P=\left(\sigma_{x x}+\sigma_{y y}+\sigma_{z z}\right) / 3, \sigma_{2}=\sqrt{3}\left(\sigma_{x x}-\sigma_{y y}\right), \\
\sigma_{3}=2 \sigma_{z z}-\sigma_{y y}-\sigma_{x x}, \quad \sigma_{4}=\sigma_{y z}, \quad \sigma_{5}=\sigma_{x z}, \quad \sigma_{6}=\sigma_{x y},
\end{gathered}
$$

$\sigma_{i k}$ are stress tensor components, $P$ is pressure.

To clarify the use of the Gibbs potential for the description of MTs the following remarks about the free energy, Equation 4, are pertinent.

First, the equilibrium strain values satisfy the extremum conditions $\partial G / \partial u_{\alpha}=0$, which are equivalent to the conditions $\partial F / \partial u_{\alpha}=0$ if $\sigma_{\alpha}=0$. The subscripts $\alpha=1,2,3$ and $\alpha=4,5,6$ correspond to the diagonal and non-diagonal stress/strain tensor components, respectively. It is of importance that the terms linear in $u_{\alpha}$ values with $\alpha=4,5,6$ are absent in the Equation 4 for the free energy. It is easy to see that the equilibrium states with $u_{2}, u_{3} \neq 0, u_{4}=u_{5}=u_{6}=0$ may exist in this case. These states can have the tetragonal symmetry and the values $u_{2}, u_{3}$ form the two-component order parameter of the cubic-tetragonal MT $[39,40]$. The terms linear in $u_{\alpha}$ with $\alpha=2,3$ are present in Equation 4 , but they vanish if $u_{4}=u_{5}=u_{6}$. Due to this, the rhombohedral state with $u_{4}=u_{5}=u_{6} \neq 0, u_{1} \neq 0$, $u_{2}=u_{3}=0$ may exist [28]. In the presence of the terms of $u_{2,3} u_{4-6}^{n}$ type $(n=1,2,3)$ the cubic-tetragonal MT is not possible, because the extremum conditions for the Gibbs potential show that in the course of phase transformation characterized by the two-component order parameter $u_{2,3}$ the concomitant nondiagonal strains $u_{4-6} \sim u_{2,3}^{n}$ must appear. The appearance of these strains leads to the appropriate lowering of tetragonal symmetry of the product phase. In presence of the energy terms of $u_{4-6} u_{2,3}^{n}$ type the phase transformation characterized by the three-component order parameter is accompanied by the appearance of the concomitant strains $u_{2,3} \sim u_{4-6}^{n}$ and the appropriate lowering of rhombohedral symmetry of the product phase.

Second, the series Equation 4 involves the terms of $u_{1} u_{\alpha}^{n}$ type. As so, the cubic-tetragonal phase transition is characterized by the appearance of the concomitant component $u_{1} \sim u_{2}^{2}+u_{3}^{2}$, while the concomitant component $u_{1} \sim u_{4}^{2}+u_{5}^{2}+u_{6}^{2}$ arises in the course of the cubic-rhombohedral phase transition. The role of concomitant components is especially important for the purview of this article, because the value of $3 u_{1}$ is the trace of strain tensor, and therefore, it is related to the volume change of the alloy as

$$
3 u_{1}=v_{M T}(T)+v(P),
$$

where $v_{M T}(T)$ is the volume change that accompanies MT of the unstressed alloy and $v(P)$ is the volume change under the pressure. According to Equations 6-8 the mathematical expression for the Gibbs potential involves the term $P v$, which is linear in both volume change and pressure. If the 
Helmholtz free energy involves the bilinear combination $k X v$ of some physical value $X$ and volume change $v$ (here $k$ is dimensionless constant), the combination $k X$ can be considered as an "internal" pressure acting on the ideal cubic lattice. The crystal defects, impurities and spatially ordered spins or dipole moments of atoms are the most important sources of the internal pressure.

Finally, it is of importance that the Equation 4 involves the third-order terms in the order parameters components (the coefficients of these terms are $a_{4}$ and $a_{5}$ ). As so, all MTs are the first-order phase transitions (see [41] and references therein). The detailed consideration of the role of third-order energy terms in the transformational behavior of alloys will be presented in Section 6.2.

\subsection{Cubic-Tetragonal MT}

In the case of cubic-tetragonal MT the non-diagonal strain tensor components $u_{4}, u_{5}$ and $u_{6}$ are equal to zero in both parent and product phases. The tendency to volume conservation during MT is observed for all phase transformations of martensitic type. This tendency is expressed by strong inequality $u_{1}^{2}<<u_{2}^{2}+u_{3}^{2}$ and therefore, the energy terms $b_{1} u_{1}^{4}, b_{2} u_{1}^{2}\left(u_{2}^{2}+u_{3}^{2}\right)$ and $a_{1} u_{1}^{3}$ can be considered small as compared with $b_{4}\left(u_{2}^{2}+u_{3}^{2}\right)^{2}$ and $a_{2} u_{1}\left(u_{2}^{2}+u_{3}^{2}\right)$. In that case the equation for the Gibbs potential takes the following form

$$
\begin{aligned}
G= & \frac{1}{2} c_{1} u_{1}^{2}+\frac{1}{2} c_{2}\left(u_{2}^{2}+u_{3}^{2}\right)+\frac{1}{2} a_{2} u_{1}\left(u_{2}^{2}+u_{3}^{2}\right)+\frac{1}{3} a_{4} u_{3}\left(u_{3}^{2}-3 u_{2}^{2}\right) \\
& +\frac{1}{4} b_{4}\left(u_{2}^{2}+u_{3}^{2}\right)^{2}+\frac{1}{2} b_{7} u_{1} u_{3}\left(u_{3}^{2}-3 u_{2}^{2}\right)+3 P u_{1}-\frac{1}{6}\left(\sigma_{2} u_{2}+\sigma_{3} u_{3}\right) .
\end{aligned}
$$

The nondiagonal stress components are not conjugated with the order parameter of cubic-tetragonal MT and are omitted in Equation 9 hence. The coefficient $c_{2}(T)$ changes sign when the cubic-tetragonal MT occurs. Due to this, the cubic-tetragonal MT is accompanied by a pronounced softening of the elastic modulus $C^{\prime}$, see Equation 5. The volume change that accompanies cubic-tetragonal MT can be interrelated with the order parameter components. The condition $\partial G / \partial u_{1}=0$ results in the following relationship:

$$
u_{1}=-\left[a_{2}\left(u_{2}^{2}+u_{3}^{2}\right)+b_{7} u_{3}\left(u_{3}^{2}-3 u_{2}^{2}\right)\right] / 2 c_{1}-\left(3 P / c_{1}\right) .
$$

Using Equation 10, the $u_{1}$ value can be excluded from Equation 9 and the Gibbs potential can be expressed through the order parameter components as

$$
G=\frac{1}{2} c_{2}^{*}(T, P)\left(u_{2}^{2}+u_{3}^{2}\right)+\frac{1}{3} a_{4}^{*}(P) u_{3}\left(u_{3}^{2}-3 u_{2}^{2}\right)+\frac{1}{4} b_{4}^{*}\left(u_{2}^{2}+u_{3}^{2}\right)^{2}-\frac{1}{6}\left(\sigma_{2} u_{2}+\sigma_{3} u_{3}\right),
$$

where

$$
\begin{aligned}
& c_{2}^{*}(T, P)=c_{2}(T)-3 a_{2} P / c_{1}, \\
& a_{4}^{*}(P)=a_{4}-9 \Omega a_{2} P / 2 c_{1}, \\
& b_{4}^{*}=b_{4}-a_{2}^{2} / 2 c_{1},
\end{aligned}
$$

and the dimensionless parameter $\Omega=b_{7} / a_{2}$ are introduced.

The expansion of the Gibbs potential in terms of the order parameter components (Equation 11) enables the description of the cubic-tetragonal MT in the framework of Landau theory of phase transitions. The conditions $\partial G / \partial u_{2}=\partial G / \partial u_{3}=0$ result in the equations describing the dependencies 
of the order parameter components on the temperature and pressure. A zero solution of these equations corresponds to the high-temperature (cubic) phase. The nonzero solutions correspond to three equivalent variants of tetragonal lattice, which are usually referred to as the $x$ - $y$ - and $z$-variants of martensitic phase. The principal axis ( $c$-axis) of $x$-variant is parallel to the crystallographic direction [100] of cubic phase, the principal axes of $y$ - and $z$-variants are aligned with [010] and [001] directions, respectively (for more details see $[39,40]$ ).

For the sake of definiteness, let us consider the transformation of cubic phase into $z$-variant of tetragonal phase occurring in the absence of anisotropic stressing (i.e., in the case of $\sigma_{2}=\sigma_{3}=0$ ). For this variant of martensitic phase $\varepsilon_{x x}=\varepsilon_{y y}$ and therefore, $u_{2}(T, P)=0$ in both austenitic and martensitic phases. The solution of the equation

$$
\partial G / \partial u_{3}=u_{3}\left[c_{2}^{*}(T, P)+a_{4}^{*}(P) u_{3}+b_{4}^{*} u_{3}^{2}\right]=0
$$

is

$$
u_{3}(T, P)= \begin{cases}0, & \text { in the cubic phase } \\ u_{0}, & \text { in the tetragonal phase }\end{cases}
$$

where

$$
\begin{aligned}
& u_{0}(T, P)=-\frac{a_{4}^{*}(P)}{2 b_{4}^{*}}\left[1+\sqrt{1-c_{2}^{*}(T, P) / c_{t}(P)}\right], \\
& c_{t}(P)=\left[a_{4}^{*}(P)\right]^{2} / 4 b_{4}^{*}>0 .
\end{aligned}
$$

As far as the volume change $v_{M T}$ is substantially smaller than the equilibrium value $u_{0}$ of the order parameter $u_{3}$, an approximate equality $\varepsilon_{x x}+\varepsilon_{y y} \approx-2 \varepsilon_{z z}$ is held. Due to this, the equilibrium value of the order parameter can be evaluated from the experimental values of lattice parameters of cubic and tetragonal phases ( $a_{0}$ and $a, c$, respectively) using the relationships

$$
u_{0} \approx 3 \varepsilon_{M}=3\left(c-a_{0}\right) / a_{0}=2(1-c / a),
$$

(see Equations 1 and 2). Here the value $\varepsilon_{M} \equiv \varepsilon_{[001]}$ is the specific elongation/contraction of the unit cell of cubic lattice caused by MT; this value is referred to as the martensitic transformation strain.

In accordance with the general principles of Landau theory, the high-temperature (austenitic) phase is stable if the coefficient of the second-order energy term is positive, that is if $c_{2}^{*}(T, P)>0$. The solution describing the low-temperature (martensitic) phase is stable if $c_{2}^{*}(T, P)<c_{t}(P)$. If the coefficients, Equation 12, are known, the equations

$$
c_{2}^{*}\left(T_{2}, P\right)=0, \quad c_{2}^{*}\left(T_{1}, P\right)=c_{t}(P),
$$

enable determination of the lability lines $T=T_{2}(P)$ and $T=T_{1}(P)$ for the austenitic and martensitic phases, respectively. In view that the cubic-tetragonal phase transformations (forward MTs) are observed on cooling, the energy coefficient $c_{2}^{*}$ is the increasing function of temperature, so the inequality $T_{1}>T_{2}$ is held.

It should be emphasized that Equations 15-17 are suitable for a description of the transformational and deformational properties of the imperfect samples of paramagnetic and magnetically ordered shape memory alloys. Both defect formation/reconfiguration and magnetic ordering of the alloy results in the 
volume change, and therefore, induces an "internal" pressure. The pressure caused by the defects formation/reconfiguration is proportional to their concentration; the pressure that is induced due to the magnetic ordering is strictly related to the volume magnetostriction of an alloy.

For the quantitative description of the cubic-tetragonal MT, the coefficients of the Landau expansion for Gibbs potential (Equation 11) must be expressed through the basic measurable characteristics of SMA. For this purpose the relationships

$$
\begin{aligned}
& u_{0}\left(T_{2}, P\right)=-2\left[1-c\left(T_{2}, P\right) / a\left(T_{2}, P\right)\right]=-a_{4}^{*}(P) / b_{4}^{*}, \\
& c_{2}^{*}\left(T_{1}, P\right)=C^{\prime}\left(T_{1}, P\right) / 3=\left[a_{4}^{*}(P)\right]^{2} / 4 b_{4}^{*}
\end{aligned}
$$

can be used. These relationships show that

$$
\begin{aligned}
& a_{4}^{*}(P)=-\frac{4 c_{2}^{*}\left(T_{1}, P\right)}{u_{0}\left(T_{2}, P\right)}=\frac{2}{3} \cdot \frac{C^{\prime}\left(T_{1}, P\right)}{1-c\left(T_{2}, P\right) / a\left(T_{2}, P\right)}, \\
& b_{4}^{*}=-\frac{a_{4}^{*}(P)}{u_{0}\left(T_{2}, P\right)}=\frac{1}{3} \cdot \frac{C^{\prime}\left(T_{1}, P\right)}{\left[1-c\left(T_{2}, P\right) / a\left(T_{2}, P\right)\right]^{2}},
\end{aligned}
$$

where $a\left(T_{2}, P\right), c\left(T_{2}, P\right)$ are the lattice parameters of tetragonal lattice.

In accordance with Equation 12, the $b_{4}^{*}$ value is independent of pressure. As so, the Equation 19 predicts that the shear modulus of cubic phase measured just before the start of MT depends on the pressure as the second power of MT strain measured just after the finish of MT. This prediction can be verified experimentally.

The expression

$$
v_{M T}=-\frac{9 a_{2} \varepsilon_{M}^{2}\left(T_{2}, 0\right)\left[1+3 \Omega \varepsilon_{M}\left(T_{2}, 0\right)\right]}{2\left(C_{11}+2 C_{12}\right)},
$$

which follows from Equation 10, establishes the interrelation between the MT strain and volume change during MT. However, this interdependence involves the undetermined parameters $a_{2}$ and $\Omega$. It may be assumed that all third-order and fourth-order energy coefficients are in roughly the same ratio to each other. This assumption results in the estimation: $|\Omega|=\left|b_{7} / a_{2}\right| \sim\left|b_{4} / a_{4}\right| \sim\left|u_{0}^{-1}\right|=\left|3 \varepsilon_{M}\right|^{-1}$. In this case the order of magnitude of the energy coefficient $a_{2}$ can be determined from the approximate relationship

$$
\left|a_{2}\right| \sim-\frac{1}{9} \cdot \frac{v_{M T}\left(C_{11}+2 C_{12}\right)}{\varepsilon_{M}^{2}\left(T_{2}, 0\right)},
$$

where the values $C_{11}, C_{12}, \varepsilon_{M}$, and $v_{M T}<0$ can be determined from the experiments.

\subsection{Cubic-Rhombohedral MT}

The cubic-rhombohedral MT is accompanied by the appearance of non-zero values of shear strains $u_{4}, u_{5}, u_{6}$ while the diagonal strain tensor components $u_{2}$ and $u_{3}$ are equal to zero in both cubic and rhombohedral phases. The tendency to volume conservation during MT is expressed by inequality $u_{1}^{2}<<u_{4}^{2}+u_{5}^{2}+u_{6}^{2}$. Due to this, the energy terms $b_{1} u_{1}^{4}, b_{3} u_{1}^{2}\left(u_{4}^{2}+u_{5}^{2}+u_{6}^{2}\right)$ and $a_{1} u_{1}^{3}$ can be 
considered negligibly small as compared with $b_{5}\left(u_{4}^{2}+u_{5}^{2}+u_{6}^{2}\right)^{2}$ and $a_{3} u_{1}\left(u_{4}^{2}+u_{5}^{2}+u_{6}^{2}\right)$, respectively. In this case the expression for the Gibbs potential, Equation 4 , is reduced to the form

$$
\begin{aligned}
G & =\frac{1}{2} c_{1} u_{1}^{2}+\frac{1}{2} c_{3}\left(u_{4}^{2}+u_{5}^{2}+u_{6}^{2}\right)+\frac{1}{2} a_{3} u_{1}\left(u_{4}^{2}+u_{5}^{2}+u_{6}^{2}\right)+a_{5} u_{4} u_{5} u_{6}+\frac{1}{4} b_{5}\left(u_{4}^{2}+u_{5}^{2}+u_{6}^{2}\right)^{2} \\
& +b_{8} u_{1} u_{4} u_{5} u_{6}+\frac{1}{4} b_{10}\left(u_{4}^{4}+u_{5}^{4}+u_{4}^{4}\right)+3 P u_{1}-2\left(\sigma_{4} u_{4}+\sigma_{5} u_{5}+\sigma_{6} u_{6}\right) .
\end{aligned}
$$

The coefficient $c_{3}(T)$ changes sign when the cubic-rhombohedral MT occurs. This coefficient is proportional to the shear modulus $C_{44}$ in the cubic phase (Equation 5), and therefore, the cubic-rhombohedral MT is accompanied by the pronounced softening of this modulus. The extremum condition for the Gibbs potential $\partial G / \partial u_{1}=0$ establishes the following interrelation between the volume change and order parameter components:

$$
v_{M T} / 3 \equiv u_{1}=-a_{3}\left(u_{4}^{2}+u_{5}^{2}+u_{6}^{2}\right) / 2 c_{1}-\left(b_{8} u_{4} u_{5} u_{6}+3 P\right) / c_{1} .
$$

The substitution of the $u_{1}$ value (Equation 23) into Equation 22 results in the following Landau expansion for the Gibbs potential

$$
\begin{aligned}
G= & \frac{1}{2} c_{3}^{*}(T, P)\left(u_{4}^{2}+u_{5}^{2}+u_{6}^{2}\right)+a_{5}^{*}(P) u_{4} u_{5} u_{6}+\frac{1}{4} b_{5}^{*}\left(u_{4}^{2}+u_{5}^{2}+u_{6}^{2}\right)^{2} \\
& +\frac{1}{4} b_{10}\left(u_{4}^{4}+u_{5}^{4}+u_{4}^{4}\right)-2\left(\sigma_{4} u_{4}+\sigma_{5} u_{5}+\sigma_{6} u_{6}\right),
\end{aligned}
$$

where

$$
\begin{aligned}
& c_{3}^{*}(T, P)=c_{3}(T)-3 a_{3} P / c_{1}, \\
& a_{5}^{*}(P)=a_{5}-3 \Lambda a_{3} P / c_{1}, \\
& b_{5}^{*}=b_{5}-a_{3}^{2} / 2 c_{1},
\end{aligned}
$$

and the dimensionless parameter $\Lambda=b_{8} / a_{3}$ is introduced.

The dependencies of the order parameter components on the temperature and pressure values obey the equations $\partial G / \partial u_{4-6}=0$. A zero solution of these equations corresponds to the high-temperature phase. The nonzero solutions correspond to the four equivalent variants of rhombohedral lattice with

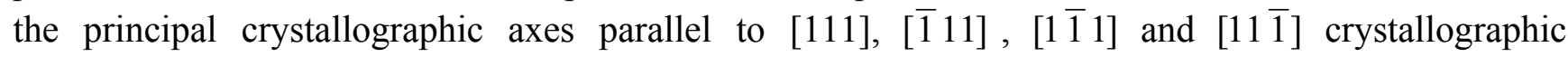
directions (for more details see, e.g., [28]). For the sake of definiteness, let us consider the transformation of cubic phase into the [111]-variant of rhombohedral lattice occurring in the absence of anisotropic stressing (i.e., at $\sigma_{4-6}=0$ ). For [111]-variant of rhombohedral lattice $u_{4}=u_{5}=u_{6} \equiv u_{R}$. The solution of the equation

$$
\partial G / \partial u_{R}=3 u_{R}\left[c_{3}^{*}(T, P)+a_{5}^{*}(P) u_{R}+\left(3 b_{5}^{*}+b_{10}\right) u_{R}^{2}\right]=0
$$

is

$$
u_{R}(T, P)= \begin{cases}0, & \text { for cubic phase } \\ u_{0}, & \text { for rhombohedral phase }\end{cases}
$$

where 


$$
\begin{aligned}
& u_{0}(T, P)=-\frac{a_{5}^{*}(P)}{2 b^{*}}\left[1+\sqrt{1-c_{3}^{*}(T, P) / c_{R}(P)}\right], \\
& c_{R}(P)=\left[a_{5}^{*}(P)\right]^{2} / 4 b^{*}>0, \\
& b^{*}=3 b_{5}^{*}+b_{10} .
\end{aligned}
$$

An equilibrium value of the order parameter is related to the specific elongation/contraction of the cubic unit cell in [111] direction as

$$
2 u_{0}=\varepsilon_{[111]} \equiv \varepsilon_{M} .
$$

The high-temperature phase is stable if $c_{3}^{*}(T, P)>0$. The solution describing the low-temperature phase is stable if $c_{3}^{*}(T, P)<c_{R}(P)$. In the case when the Landau expansion coefficients are determined from the equations Equation 25, the conditions

$$
c_{3}^{*}\left(T_{2}, P\right)=0, \quad c_{3}^{*}\left(T_{1}, P\right)=c_{R}(P),
$$

enable determination of the lability lines $T=T_{2}(P)$ and $T=T_{1}(P)$ for the austenitic and martensitic phases, respectively. The cubic-rhombohedral phase transformations (forward MTs) are observed on cooling of the alloys, so the energy coefficient $c_{3}^{*}$ is the increasing function of temperature and the inequality $T_{1}>T_{2}$ holds.

The Equations 27-30 are suitable for description of the transformational and deformational properties of the imperfect paramagnetic and magnetically ordered shape memory alloys. The coefficients of Equation 25 in the Landau expansion for the Gibbs potential depend on the effective pressure that may be induced by the hydrostatic load, defects formation/reconfiguration, magnetic ordering of SMA, etc. For the quantitative description of the cubic-rhombohedral MT, these coefficients must be expressed through the basic measurable characteristics of SMA. The relationships

$$
\begin{aligned}
& u_{0}\left(T_{2}, P\right)=\varepsilon_{M}\left(T_{2}, P\right) / 2=-a_{5}^{*}(P) / b^{*}, \\
& c_{3}^{*}\left(T_{1}, P\right)=4 C_{44}\left(T_{1}, P\right)=\left[a_{5}^{*}(P)\right]^{2} / 4 b^{*}
\end{aligned}
$$

result in the expressions

$$
\begin{aligned}
& a_{5}^{*}(P)=-\frac{4 c_{3}^{*}\left(T_{1}, P\right)}{u_{0}\left(T_{2}, P\right)}=-32 \cdot \frac{C_{44}\left(T_{1}, P\right)}{\varepsilon_{[111]}\left(T_{2}, P\right)}, \\
& b^{*}=-\frac{a_{5}^{*}(P)}{u_{0}\left(T_{2}, P\right)}=64 \cdot \frac{C_{44}\left(T_{1}, P\right)}{\varepsilon_{[111]}^{2}\left(T_{2}, P\right)} .
\end{aligned}
$$

In accordance with Equation 25, the $b^{*}$ value is independent of pressure and hence Equation 32 predicts that the elastic modulus $C_{44}$ of cubic phase measured just before the start of MT depends on the pressure as the second power of MT strain measured just after the finish of MT. The interdependence between the soft elastic modulus and MT strain can be verified in the experiments with the different alloys belonging to the same alloy family.

The expression

$$
v_{M T}=-\frac{3 a_{3} \varepsilon_{[111]}^{2}\left(T_{2}, P\right)\left[1+\Lambda \varepsilon_{[111]}\left(T_{2}, P\right) / 3\right]}{8\left(C_{11}+2 C_{12}\right)},
$$


which follows from Equation 23, describes the interrelation between the order parameter and volume change of MT. The Equation 33 involves the undetermined parameters $a_{3}$ and $\Lambda$.

If all third-order and fourth-order energy coefficients are in roughly the same ratio to each other, the estimation $|\Lambda|=\left|b_{8} / a_{3}\right| \sim\left|b^{*} / a_{5}^{*}\right| \sim\left|u_{0}^{-1}\right|=2\left|\mathcal{E}_{[111]}\right|^{-1}$ is valid and the energy coefficient $a_{3}$ can be estimated by the order of magnitude from the relationship

$$
\left|a_{3}\right| \sim-\frac{v_{M T}\left(C_{11}+2 C_{12}\right)}{\varepsilon_{[111]}^{2}\left(T_{2}, 0\right)},
$$

which involves the measurable values $C_{11}, C_{12}, \varepsilon_{M}$, and $v_{M T}<0$.

\subsection{Summary of Basic Points and Preview of Applications of the Theory}

Summarizing the main points of the symmetry conforming theory of MTs in the SMAs, we can conclude that this theory starts from the invariant (with respect to the cubic symmetry group) expression for the Gibbs potential. This expression can be represented as the Landau expansion in the order parameter components (see Equations 11 and 24). The expansion coefficients depend on the pressure produced by the hydrostatic mechanical load or on the "internal" pressure caused by crystallographic defects formation, chemical or magnetic ordering of the alloy, and any other physical factor, which changes the volume of SMA.

Formally, the pressure results in the renormalization of the Landau expansion coefficients. It is especially important for the purview of this article that the renormalization of the coefficients $c_{2}(T)$ and $c_{3}(T)$ of the second-order energy terms is proportional to (i) pressure value and (ii) the ratio of volume change during MT to the squared MT strain, that is

$$
c_{2,3}^{*}-c_{2,3} \propto\left(v_{M T} / \varepsilon_{M}^{2}\right) P .
$$

This follows from Equations 12 and 21 for $c_{2}$ and Equations 25 and 34 for $c_{3}$. In this line of reasoning, all physical effects, which can be described in terms of the internal pressure, must be closely related to the volume changes that accompany MTs.

The drastic influence of the volume change on the elastic, thermodynamic and magnetic properties of SMAs will be demonstrated in the next sections of this overview. Special attention will be paid to the effects caused by hydrostatic loading, martensite aging and cyclic across forward/reverse MTs. The physical consequences of spontaneous volume magnetostriction of ferromagnetic SMAs will be also described. The Landau expansion coefficients will be estimated for the representative Ti-Ni, Au-Cd and Ni-Mn-Ga alloys. The interdependence between the volume changes caused by the MTs in these alloys, on the one hand, and MT temperatures, MT strains and elastic modules, on the other hand, will be studied. The contribution of volume magnetostriction to the entropy change during MT of ferromagnetic shape memory alloy will be disclosed by consideration of the representative Ni-Mn-Ga and Ni-Fe-Ga alloys.

It is commonly known that the cubic-orthorhombic or cubic-monoclinic MTs are observed in many alloys. In some cases the product phases arising in the course of these MTs are characterized by the slightly distorted tetragonal or rhombohedral crystallographic cells. In these cases Equation 35 approximately relates the volume change to the main component of MT strain. It may be 
expected therefore that the conclusions derived from this equation are valid for the majority of thermoelastic alloys.

\section{Martensite Stabilization/Destabilization under Hydrostatic Pressure}

\subsection{Interrelation between the Volume Change during MT and Pressure Effects}

The hydrostatic compression stabilizes the martensitic phase if the volume of alloy decreases during the forward MT (i.e., if $v_{M T}<0$ ) and destabilizes it otherwise. The stabilization/destabilization of martensitic phase under pressure manifests itself basically as

a) an increase/decrease of the characteristic MT temperatures [24-26,42,43];

b) an increase/decrease of the soft elastic modulus of martensite.

If the transformation goes in the quasiequilibrium way, the shifts of martensite start and martensite finish temperatures under pressure are approximately equal to the shifts of the lability temperatures of the martensitic and austenitic phases $T_{1}$ and $T_{2}$. In the simplest approach, the influence of pressure on MT can be characterized by the change of the average temperature $T_{0} \equiv\left(T_{1}+T_{2}\right) / 2$. This change proves to be strictly proportional to the pressure value $[25,26]$ and the coefficient of proportionality $d T_{0} / d P$ can be estimated from the Clausius-Clapeyron relationship $d T_{0} / d P=v_{M T} T_{0} / q m$, where $m$ is mass density, $q$ is the latent heat of MT normalized to the unit of mass. Hence, the shift of MT temperature under hydrostatic pressure is expressed as

$$
\Delta T=\left(\frac{v_{M T} T_{0}}{q m}\right) P .
$$

The MT of cubic-tetragonal or cubic-rhombohedral type is accompanied by the pronounced softening of the shear elastic modulus $C^{\prime}$ or $C_{44}$, respectively. The pressure influence on the value of shear modulus in the austenitic phase of alloy can be estimated using the relationship

$$
\Delta C^{\prime} \approx \frac{\partial C^{\prime}}{\partial P} P \sim\left(\frac{v_{M T}}{3 \varepsilon_{M}^{2}}\right) P
$$

or

$$
\Delta C_{44} \approx \frac{\partial C_{44}}{\partial P} P \sim\left(\frac{v_{M T}}{4 \varepsilon_{M}^{2}}\right) P,
$$

which follows from Equations 12,21,25,34, respectively. One can see now that the influence of pressure on the MT temperature and shear modulus of an alloy is proportional to the product $P v_{M T}$. This result is in agreement with Equation 35 and general conclusion derived from this equation in the Section 2.4.

Every physical factor, which causes the martensite stabilization, noticeably changes the functional properties of SMAs. In the particular case of martensite stabilization by alloy aging, this statement is confirmed by the numerous experiments (see [44-47] and references therein). To extend this statement to include the other factors of martensite stabilization, the influence of hydrostatic pressure on the MT strain and shear elastic modulus of SMA will be considered below. The Ti-Ni alloy system will be 
chosen as the illustrative example. The properties of Ti-Ni alloys deserve a consideration because of the broad use of these alloys in the medicine [6] and engineering [4,5,7].

\subsection{Landau Expansion Coefficients Estimated from the Shear Modulus of Ti-Ni Alloy}

The Ti-Ni alloys exhibit both cubic-monoclinic $(\mathrm{B} 2 \rightarrow \mathrm{B} 19)$ and cubic-rhombohedral $(\mathrm{B} 2 \rightarrow \mathrm{R})$ transformations [48]. A consistent theory of the cubic-monoclinic phase transformation is not developed yet, while the cubic-rhombohedral transformation can be studied using Equations 26-30. The coefficients of Equation 26 can be estimated from the shear modulus $C_{44}^{(A)}$, which describes the elastic response of alloy in the austenitic state to the mechanical loading in [111] crystallographic direction. In the presence of stress $\sigma \equiv \sigma_{[111]}$, Equation 26 is modified as

$$
u_{R}\left[c_{3}^{*}(T, P)+a_{5}^{*}(P) u_{R}+b^{*} u_{R}^{2}\right]-2 \sigma=0 .
$$

The solution of Equation 39 can be presented as a sum of the spontaneous shear strain $u_{0}$ (Equation 27) and elastic strain $\tilde{u}_{R}$. The substitution of the values $u_{R}=u_{0}(T, P)+\tilde{u}_{R}$ and $c_{3}^{*}(T, P)=4 C_{44}^{(A)}$ into Equation 39 leads to the formula that interrelates the MT strain and the difference of shear modules characterizing the martensitic and austenitic states of an alloy:

$$
C_{44}^{(M)}(T, P)-C_{44}^{(A)}(T, P)=\frac{1}{2} a_{5}^{*}(P) u_{0}(T, P)+\frac{3}{4} b^{*} u_{0}^{2}(T, P)
$$

It is quite natural that the difference of the elastic modules of two phases tends to zero in the limiting case $u_{0} \rightarrow 0$, because in this case the phases become physically equivalent. Using Equation 26, one can express the elastic modulus of martensitic phase as

$$
C_{44}^{(M)}(T, P)=\frac{1}{4} a_{5}^{*}(P) u_{0}(T, P)+\frac{1}{2} b^{*} u_{0}^{2}(T, P) .
$$

Substituting $u_{0}\left(T_{2}, P\right)=-a_{5}^{*}(P) / b^{*}$ (see Equation 31) into Equation 41, one can derive the relationships

$$
C_{44}^{(M)}\left(T_{2}, P\right)=4 C_{44}^{(A)}\left(T_{1}, P\right)
$$

and

$$
b^{*}=4 C_{44}^{(M)}\left(T_{2}, P\right) / u_{0}^{2}\left(T_{2}, P\right) .
$$

The Equations 28,39,42,43 enable the solution of two following problems:

(i) a theoretical evaluation of the shear modulus from the experimental temperature dependencies of lattice parameters in the martensitic phase;

(ii) a restoring of the temperature dependence of MT strain from the temperature dependence of the shear modulus.

An example of the solution of the first problem, the cubic-tetragonal MT was described in [49]. The solution of the second problem is presented below. 
For the sake of definiteness, let us consider the experimentally observed cubic-rhombohedral MT in $\mathrm{Ti}_{49.5} \mathrm{Ni}_{50.5}$ alloy [50]. The temperature dependence of $C_{44}$ modulus of $\mathrm{Ti}_{49.5} \mathrm{Ni}_{50.5}$ alloy measured in [50] is shown in Figure 1 by circles.

Figure 1. Experimental values of shear elastic modulus of the $\mathrm{Ti}_{49.5} \mathrm{Ni}_{50.5}$ alloy [50] (circles) and the extrapolation of experimental data used for computation of martensitic transformation (MT) strain (line). The arrows point to the characteristic MT temperatures and correspondent values of shear modulus.

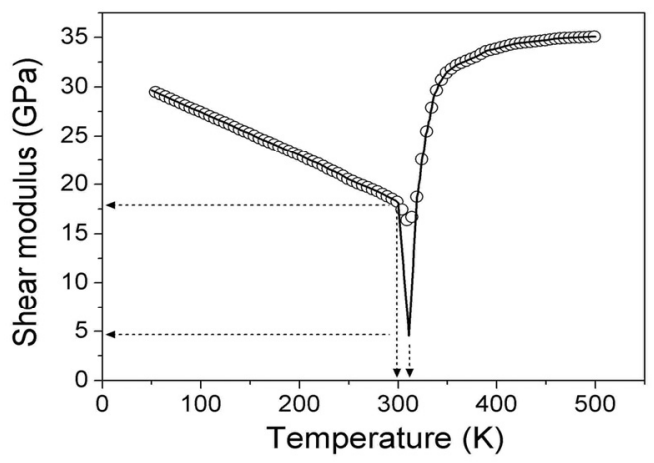

The lability temperatures of martensitic and austenitic phases derived from the experimental values of soft modulus are $T_{1}=311 \mathrm{~K}$ and $T_{2}=300 \mathrm{~K}$, respectively (see arrows in Figure 1). Moreover, the experiment shows that $C_{44}\left(T_{2}\right)=18 \mathrm{GPa}$. In this case Equation 42 results in the value $C_{44}\left(T_{1}\right)=4.5 \mathrm{GPa}$. The energy coefficients $a_{5}^{*}(0)=1.45 \times 10^{4} \mathrm{GPa}$ and $b^{*}=2.9 \times 10^{7} \mathrm{GPa}$ can be found from Equation 32 using experimental value $\varepsilon_{[111]}=2 u_{0}\left(T_{2}\right) \approx-1 \%$ [48] and estimation $v_{M T} \approx-5 \times 10^{-4}$ resulting from the Clausius-Clapeyron relationship (for more details see [28]). To describe an influence of the hydrostatic pressure, a dimensionless parameter $\Lambda$ that is involved in the renormalized energy coefficients, Equation 25 , should be estimated to be $|\Lambda| \sim 2\left|\varepsilon_{[111]}^{-1}\right| \approx 200$ (see the last paragraph of Section 2.3). The estimated values enable calculation of the ratio $a_{3} / c_{1}$ and coefficients $c_{3}(T, P), a_{5}^{*}(P)$ from Equations 33 and 25. Due to this, a quantitative description of the transformational behavior of the single-crystalline sample under the action of uniaxial mechanical loading becomes possible (the appropriate theoretical procedure is described in [49]).

\subsection{The Influence of Pressure on the MT Strain and MT Temperature}

Solving of Equation 41 with respect to $u_{0}$ value enables the quantitative description of the pressure influence on the MT strain $\varepsilon_{[111]}$ if the parameter $\Lambda$, energy coefficients $a_{5}^{*}, b^{*}$, and function $C_{44}(T, 0)$ are known. The energy coefficients were determined above for the $\mathrm{Ti}_{49.5} \mathrm{Ni}_{50.5}$ alloy. The $C_{44}(T, 0)$ function must coincide with experimental temperature dependence obtained for the austenitic and martensitic phases of this alloy and satisfy Equation 42. This function is shown in Figure 1 by the solid line. The figure demonstrates that experimental temperature dependence of shear modulus does not satisfy the condition of Equation 42. A disagreement between theory and experiment is obviously caused by the appearance of mixed two-phase state in the temperature range of $T_{2}<T<T_{1}$.

Figure 2(a) shows the solutions of Equation 41 computed for different $\Lambda$ values using the $C_{44}(T, 0)$ function shown in Figure 1 by the solid line. It is seen that the pressure of 1 GPa noticeably changes 
the temperature dependencies of the MT strain if $\Lambda=-200$ or 400 but leaves it almost unchanged if $\Lambda=200$. It means that the computations show the non-monotone dependence of MT strain on $\Lambda$ value. If $\Lambda=-200$, the theory predicts a monotone dependence of MT strain on pressure; the pressure of $2 \mathrm{GPa}$ increases the absolute value of MT strain by $25 \%$ (Figure 2(b)).

Figure 2. (a), (b) The temperature-dependent MT strains restored from the temperature dependence of soft elastic modulus.
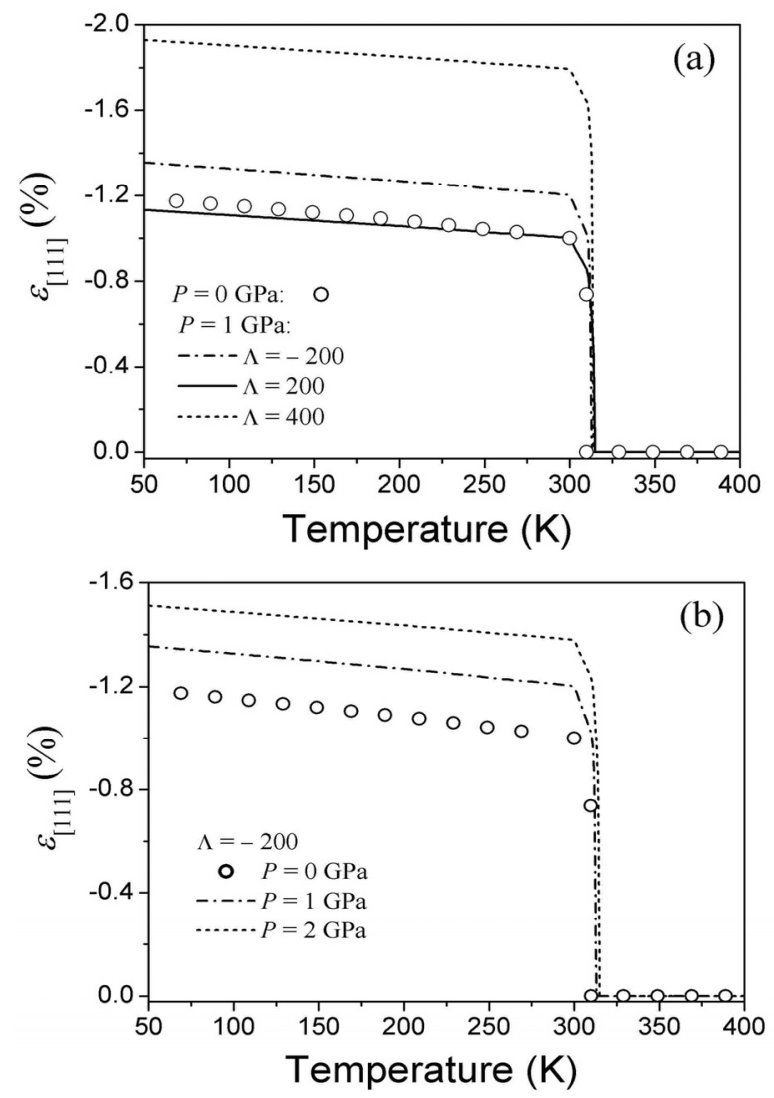

The shifts of MT temperatures caused by a hydrostatic pressure are measured in experiments (see, e.g., $[25,26,28]$ ). It is observed that the shifts of the forward and reverse MT temperatures are close in value. To describe the influence of pressure on the MT temperatures, an average value $T_{0}(P)=\left[T_{1}(P)+T_{2}(P)\right] / 2$ of lability temperatures $T_{1}(P)$ and $T_{2}(P)$ can be computed. This value is shown in Figure 3(a). It is seen that the variation of MT temperature is roughly equal to $10 \mathrm{~K} / \mathrm{GPa}$ and $2 \mathrm{~K} / \mathrm{GPa}$ for the values $\Lambda=200$ and $\Lambda=-200$, respectively. The difference $\Delta T(P)=T_{1}(P)-T_{2}(P)$ (that is the width of two-phase range) also depends on pressure (see Figure 3(b)). The pressure application diminishes the width of two-phase temperature range. This diminution is especially pronounced if the parameter $\Lambda$ is positive: in this case the width of two-phase temperature range is less than $2 \mathrm{~K}$ for the wide interval of pressures. 
Figure 3. (a) Theoretical dependence of the MT temperature $T_{0}$ on the hydrostatic pressure. (b) Theoretical dependence of two-phase temperature interval on pressure.
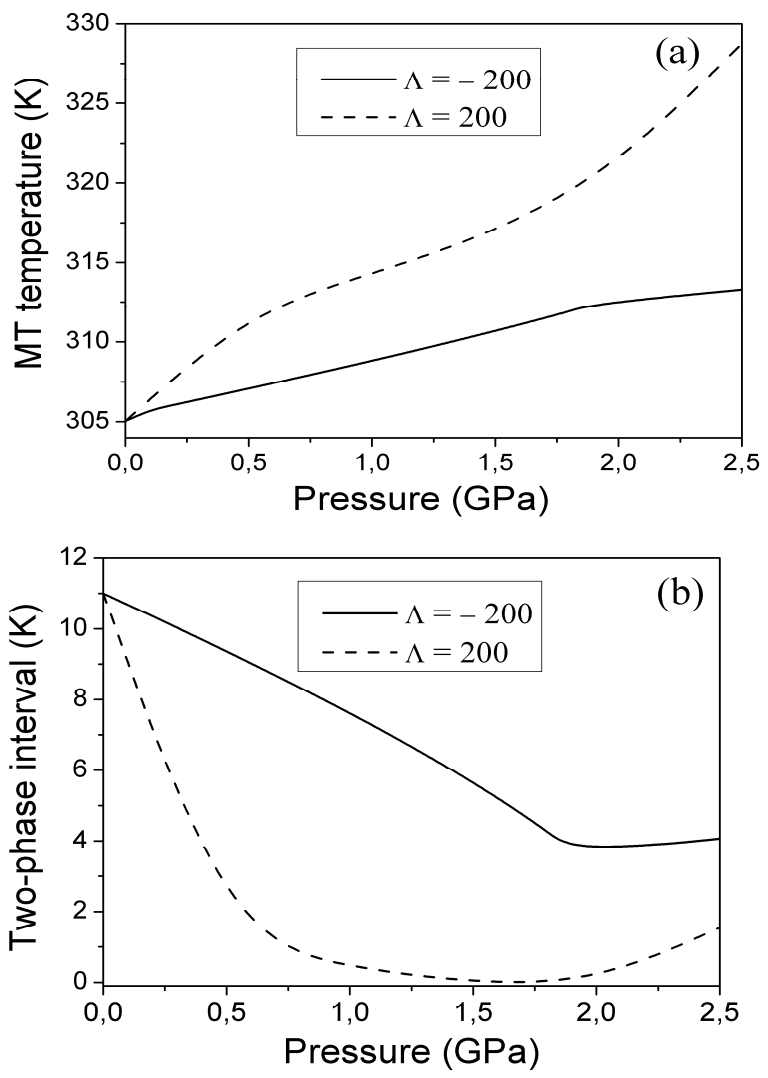

It should be remembered that the conclusions derived from Figures 2 and 3 are valid in the case of negative value of the MT strain $\varepsilon_{[111]}$. If the MT strain is positive, the sign of parameter $\Lambda$ should be changed to keep in force these conclusions.

\subsection{The Influence of Pressure on the Shear Elastic Modulus}

A measurement of the shear elastic modulus $C_{44}$ of SMA in the martensitic phase is a difficult task even in the absence of pressure. In the same time, the value of this modulus strongly affects the temperature dependence of heat capacity [51] and other thermodynamic properties of SMAs. It stipulates an importance of the calculation of the pressure and temperature dependencies of shear modulus. The results of computations are presented in Figure 4. 
Figure 4. (a) Pressure influence on temperature dependence of shear modulus computed for the negative $\Lambda$ values. (b) Pressure influence on temperature dependence of shear modulus computed for the positive $\Lambda$ values.
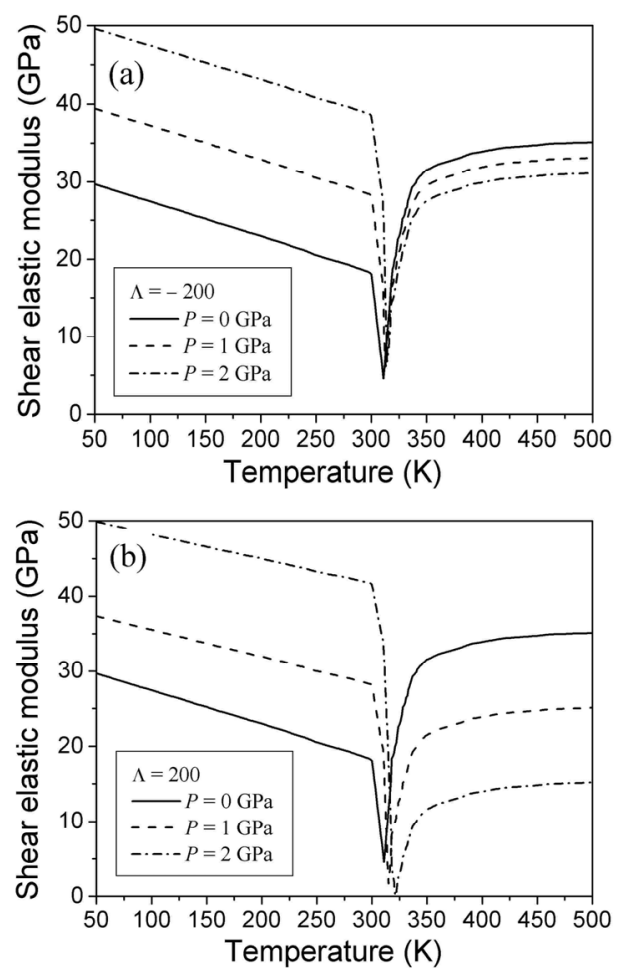

The computations show that the pressure of about $1 \mathrm{GPa}$ drastically increases the shear modulus in the martensitic phase and decreases it in the austenitic phase. This result explicitly demonstrates the stabilization of martensitic phase and the appropriate destabilization of the austenitic one. If the parameter $\Lambda$ is negative, the pressure influence on the shear elastic modulus of austenite is less pronounced than if $\Lambda>0$ but still observable, nevertheless.

It should be stressed, that in view of the ambiguity in $\Lambda$ values, an uncertainty of the theoretical results arises. However, these results are in a qualitative agreement with experimental data reported in [52]. Therefore, the strong influence of the hydrostatic pressure on the MT strains and soft elastic modules of SMAs should be expected. This influence can manifest itself, in particular, as the change of heat capacity of the alloy in the wide temperature range (see [51]).

\section{Stabilization of Martensitic Phase by Its Aging}

\subsection{Isotropic Mechanism of Martensite Stabilization}

It is commonly known that a spatial distribution of the lattice defects evolves slowly during aging of the shape memory alloys in a martensitic phase [46,53]. This evolution causes the well-pronounced physical effects, which include:

(i) a considerable difference in the stress-strain dependencies measured before and after martensite aging $[45,46,53]$;

(ii) a gradual change of the lattice parameters during martensite aging [54]; 
(iii) a martensite stabilization [44,47,55-57], that is an increase of the reverse (from martensitic to austenitic phase) MT temperature caused by martensite aging.

The works interrelating the effects of aging to the spatial symmetry of defect subsystem can be singled out from the numerous publications related to this subject [44,53,57-59]. For the particular case of the point defects, the Symmetry-Conforming Short-Range-Order (SC-SRO) principle has been formulated [53]. According to this principle, the energy of the crystal with defects is minimal when the probabilities of finding point defects in the certain crystallographic positions have the same symmetry as the crystal lattice has. A substantial generalization of SC-SRO principle follows from the special version of a Landau theory of phase transitions, which has been developed very recently for the description of interplay between the deformation of crystal lattice during MT and reconfiguration of defect subsystem [35-37]. Firstly, this theory states that the defects of any type induce the "internal" (that is existing even in the absence of external forces) mechanical stress and the energy of every (austenitic or martensitic) phase is minimal when the symmetry of stress tensor $\sigma_{i k}^{(i)}$ corresponds to the symmetry of crystal lattice in this phase. Secondly, the theory takes into account that the tendency of the crystal with defects to the ground state is accompanied by a volume change.

Stress tensor $\sigma_{i k}^{(i)}$ describes an influence of defects on the crystal lattice, and therefore, depends on time $t$ due to a slow reconfiguration of the defect subsystem during martensite aging. This tensor can be subdivided into the isotropic and axial parts as

$$
\sigma_{i k}^{(i)}(t)=P^{(i)}(t) \delta_{i k}+\sigma_{i k}^{(a x)}(t),
$$

where $\delta_{i k}$ is the Kronecker symbol, the scalar $P^{(i)}$ is interpreted as the "internal" pressure, which is responsible for the volume change accompanying the evolution of defect subsystem during the martensite aging. An axial part of the internal stress is described by the tensor $\sigma_{i k}^{(a x)}(t)$, the trace of this tensor is equal to zero.

Time-variation of the axial stress is controlled by the SC-SRO principle and is responsible for the difference in the yield stresses characterizing the stress-strain tests performed before and after the martensite aging. Moreover, the axial internal stress contributes to the martensite stabilization [35,36,60,61].

A volume change caused by the evolution of a defect subsystem after MT does not result in the symmetry change, and therefore, is an extrinsic to the SC-SRO principle. This volume change provides an additional (to symmetry conformation) physical mechanism for the martensite stabilization: if the volume changes arising during and after forward MT are of the same sign, the latter retards the reverse MT and stabilizes martensitic phase therefore. This is the isotropic mechanism of martensite stabilization $[35,36,62]$.

It was shown recently that the martensite stabilization in the representative $\mathrm{Au}-\mathrm{Cd}$ and Ni-Mn-Ga alloys is caused mainly by the isotropic mechanism [35,36]. Due to this, an evolution of the transformational properties of these alloys is satisfactorily described by the equations presented in the Sections 2 and 3, if in these equations, the hydrostatic pressure $P$ is replaced by the time-dependent internal pressure $P^{(i)}(t)$. In accordance to the arguments presented in Sections 2.4 and 3.1, an influence of the internal pressure on transformational properties of SMA is characterized by a product 
$v_{M T} \varepsilon_{M}^{-2} P^{(i)}(t)$. It means that the larger is a ratio of the volume change during MT to the MT strain (other parameters being equal), the more pronounced are aging effects.

The isotropic mechanism of a martensite stabilization can be illustrated by the consideration of martensitic transformation of Au-Cd alloy.

\subsection{Martensite Stabilization in Au-Cd Alloys}

The Au-Cd alloys exhibit MT of the cubic-rhombohedral type. For the theoretical description of martensite stabilization by aging, the Gibbs potential, Equation 24, with time-dependent coefficients

$$
\begin{aligned}
& c_{3}^{*}(T, t)=c_{3}(T)-3 a_{3} P^{(i)}(t) / c_{1}, \\
& a_{5}^{*}(t)=a_{5}-3 \Lambda a_{3} P^{(i)}(t) / c_{1}, \\
& b_{5}^{*}=b_{5}-a_{3}^{2} / 2 c_{1},
\end{aligned}
$$

should be minimized. Note that the Equations 45 are obtained from Equations 25 by the $P \rightarrow P^{(i)}(t)$ change. The time-dependence of the internal pressure can be determined from the simple physical considerations: the $P^{(i)}$ value is constant in the equilibrium state, which corresponds to the minimal energy of crystal with defects; the rate of change of $P^{(i)}$ is roughly proportional to the deviation from the equilibrium value $P^{(i)}(\infty)$, which can be formally reached after the infinite time period; a duration of the transformation cycle is small in comparison with aging time. These considerations are formalized by the equation

$$
\frac{d P^{(i)}(t)}{d t}=\frac{P^{(i)}(\infty)-P^{(i)}(t)}{\tau}
$$

where $\tau$ is characteristic time of the relaxation of defect subsystem to the equilibrium. It is convenient to count the internal pressure from its value in the "fresh" martensitic state appearing at $t=0$. In this case the internal pressure arises in the course of martensite aging and stabilizes the martensitic state. An appropriate solution of Equation 46

$$
P^{(i)}(t)=P^{(i)}(\infty)\left[1-\exp \left(-\frac{t}{\tau}\right)\right]
$$

explicitly shows that the stabilizing pressure (SP) appears immediately after MT and tends to the saturation value of $P^{(i)}(\infty)$ during a martensite aging (more formal substantiation of Equation 47 is presented in [36]).

Few thermodynamic characteristics of the Au-Cd alloy are needed for the determination of the time-dependent coefficients of the Gibbs potential, Equation 45. Unfortunately, the complete set of characteristics has never been measured for one certain alloy, and therefore, some representative alloy belonging to the $\mathrm{Au}-\mathrm{Cd}$ alloy family should be considered. The experimental values of $T_{2} \approx 300 \mathrm{~K}[63], C_{44}\left(T_{1}\right) \approx 40 \mathrm{GPa}[64]$ and $\varepsilon_{M}=2 u_{R} \approx-0.8 \%$ [65] are reported for few Au-Cd alloys and the typical value of volume change during the thermoelastic MT, $v_{M T} \approx-3 \times 10^{-3}$ [66], can be accepted. A negative value of $\Lambda=2 \varepsilon_{M}^{-1}=-250$ can be chosen (see below). With known shear modulus, the transformation strain and volume change enable us to evaluate the coefficients $a_{3} / c_{1}$, $a_{5}(0)=1.6 \times 10^{5} \mathrm{GPa}$ and $b^{*}(0)=4 \times 10^{7} \mathrm{GPa}$ from Equations 31-34. The Clausius-Clapeyron 
relationship with the values $q \approx 1 \mathrm{~J} \mathrm{~g}^{-1}$ and $m \approx 14 \mathrm{~g} \mathrm{~cm}^{-3}$ [28] results in the characteristic temperature value of $T_{1}=306.8 \mathrm{~K}$.

The values presented above prescribe a time evolution of the $c_{3}^{*}(T, t)$ coefficient whose temperature dependence leads to the phase transformation. As a consequence, the characteristic MT temperatures determined from the Equation 30 prove to be time-dependent.

The dependence of austenite start and finish temperatures on aging time $t$ can be characterized by the temperature differences $\Delta_{A S} \equiv T_{2}^{*}(t)-T_{2}^{*}(0)$ and $\Delta_{A F} \equiv T_{1}^{*}\left(t_{0}\right)-T_{1}^{*}(0)$. The value $\Delta_{12} \equiv T_{1}^{*}(t)-T_{2}^{*}(t)$ is the temperature range for the two-phase region, that represents a mixture of austenitic-martensitic structures. These temperature differences were computed using the aforementioned set of physical values and Equations 46 and 47. The relaxation time of $\tau=50 \mathrm{~min}$ and the saturation value of internal pressure $P^{(i)}(\infty)=3.8 \mathrm{GPa}$ were determined by fitting $\Delta_{A F}(t)$ theoretical curve to the experimental values reported in [57]. The results of computations are shown in Figure 5 together with the austenite finish temperature changes experimentally observed in [57].

Figure 5. Time evolution of the austenite start (dotted line), austenite finish (solid line), and two-phase temperature range (dashed-dotted line). The experimental values (circles) for the austenite finish temperature are taken from [57] and used for the evaluation of relaxation time and saturation value of stabilizing internal pressure.

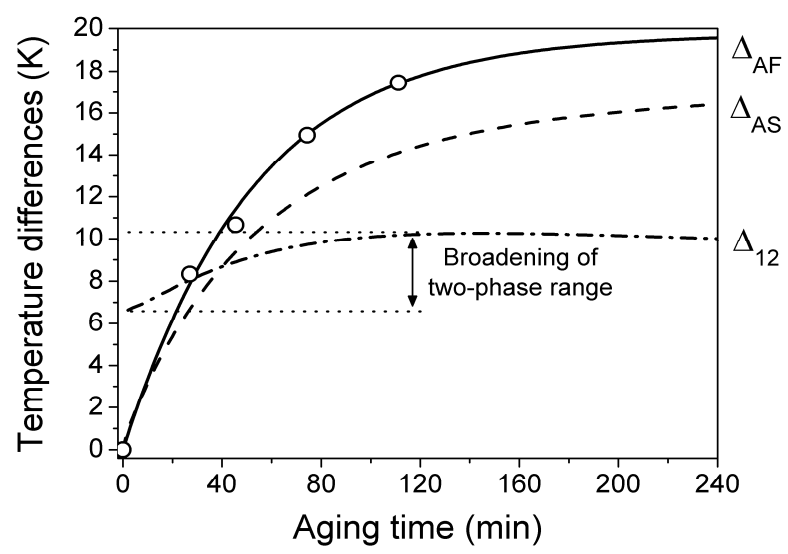

It was shown in the Section 2.3 that the absolute value of the dimensionless parameter $\Lambda$ can be estimated as $|\Lambda| \approx 2 \varepsilon_{M}^{-1}$. The negative value of $\Lambda$ was used for obtaining results presented in Figure 5 . The negative value was chosen because in this case the theory shows that the martensite aging causes broadening of the two-phase temperature range of the reverse MT (see two-side arrow in Figure 5). This feature of the transformational behavior of aged alloys is indirectly confirmed by the experiments in $[67-70]$.

The computations performed for the positive $\Lambda$ values demonstrate a narrowing of the two-phase temperature range by the martensite aging. This type of transformational behavior of an alloy is not typical but, indeed, it was observed for Co-Ni-Ga alloy [71].

It should be emphasized that the stabilizing pressure renormalizes the parameter $a_{5}^{*}$, which prescribes that MT must be the first-order phase transition. According to Equation 45 and argumentation presented in Section 2.4, we have: 


$$
a_{5}^{*}(\infty)-a_{5}(0)=-3 \Lambda a_{3} P^{(i)}(\infty) / c_{1} \sim 2 P^{(i)}(\infty) v_{M T} \varepsilon_{M}^{-3} .
$$

The values presented above result in the following estimation $\Delta a_{5}^{*} / a_{5}(0) \sim\left[0.1 \mathrm{GPa}^{-1}\right] P^{(i)}(\infty)$. This estimation shows that the jumps of physical values observed in the course of MT would be enlarged by factor of 1.4 if the stabilizing pressure of the order of 4 GPa should appear.

Let us sum up the new aspects of a martensite stabilization effect considered in $[35,36,62]$.

(i) The time evolution of the crystal defects subsystem leads to a gradual volume change of the SMA aged in the martensitic state.

(ii) According to the general principles of thermodynamics, the time-dependent internal pressure can be defined as the thermodynamic value, which is conjugated to the volume change.

(iii) The internal pressure contributes to the martensite stabilization effect but does not change the symmetry of the crystal lattice; therefore, the gradual volume change of the alloy, held in the martensitic phase, provides the isotropic mechanism of martensite stabilization, which is extrinsic to the commonly known SC-SRO principle formulated in [44,53].

(iv) The quantitative theoretical analysis of the experimental results in Ref. [57] shows that the stabilization of rhombohedral martensitic phase of Au-Cd is caused mainly by the isotropic mechanism and the corresponding value of internal pressure is about $3.5 \mathrm{GPa}$ [36].

\section{Destabilization of Martensitic Phase by Thermomechanical Cycling}

\subsection{Introductory Statement}

As we had demonstrated, the reconfiguration of defect system strongly influences the elastic and transformational properties of SMA. In particular, holding an alloy during long time in the martensitic phase leads the crystal lattice with defects to the quasiequilibrium state and causes the martensite stabilization. It suggests an idea that the cyclic thermally- or stress-induced MTs can disturb the crystal lattice with defects and move it out of an equilibrium. As so, the changes in the alloy properties, which are opposite to those described in the previous section, can be observed. This idea was confirmed in the course of cooperative (experimental and theoretical) studies of the cyclic thermally- and stress-induced MTs [37].

\subsection{Experiment}

The effect of the thermal, mechanical and combined thermomechanical cycling on the elastic properties and transformation behavior of $\mathrm{Ni}_{57.5} \mathrm{Mn}_{22.5} \mathrm{Ga}_{20.0}$ alloy has been studied [37,72]. The alloy undergoes MT from cubic phase with lattice parameter $a_{0}=0.587 \mathrm{~nm}$ to a non-modulated tetragonal phase with lattice parameters $a=0.543 \mathrm{~nm}$ and $c=0.665 \mathrm{~nm}(c / a=1.22)$.

The Sample 1 with dimensions of $0.19 \times 0.5 \times 9.1 \mathrm{~mm}^{3}$ and Sample 2 with dimensions of $0.27 \times 0.28 \times 8.5 \mathrm{~mm}^{3}$ were cut from the same $\mathrm{Ni}_{57.5} \mathrm{Mn}_{22.5} \mathrm{Ga}_{20.0}$ single crystal. Both samples were oriented in [100] crystallographic directions. The details of specimen preparation are reported in [37,72]. A TA Instruments Q800 dynamic mechanical analyzer (DMA) was used to measure: (a) the temperature dependence of the low-frequency elastic modulus in the dynamic tensile mode and (b) the tensile stress-strain curves in a quasi-static mode. 
Experimental temperature dependence of the elastic modulus of Sample 1 is shown in Figure 6 by the solid line. An abrupt change of elastic modulus in the temperature ranges of the forward $(545 \mathrm{~K}<T<575 \mathrm{~K})$ and reverse $(585 \mathrm{~K}<T<625 \mathrm{~K}) \mathrm{MTs}$ is observed. The horizontal arrows in Figure 6 illustrate that the modulus measured at martensite finish temperature $T_{M F}=545 \mathrm{~K}$ or austenite start temperature $T_{A S}=585 \mathrm{~K}$ is four times as large as that measured at martensite start temperature $T_{M S}=575 \mathrm{~K}$ or austenite finish temperature $T_{A F}=625 \mathrm{~K}$, respectively.

Figure 6. Elastic modulus of Sample 1 of $\mathrm{Ni}_{57.5} \mathrm{Mn}_{22.5} \mathrm{Ga}_{20.0}$ single crystal.

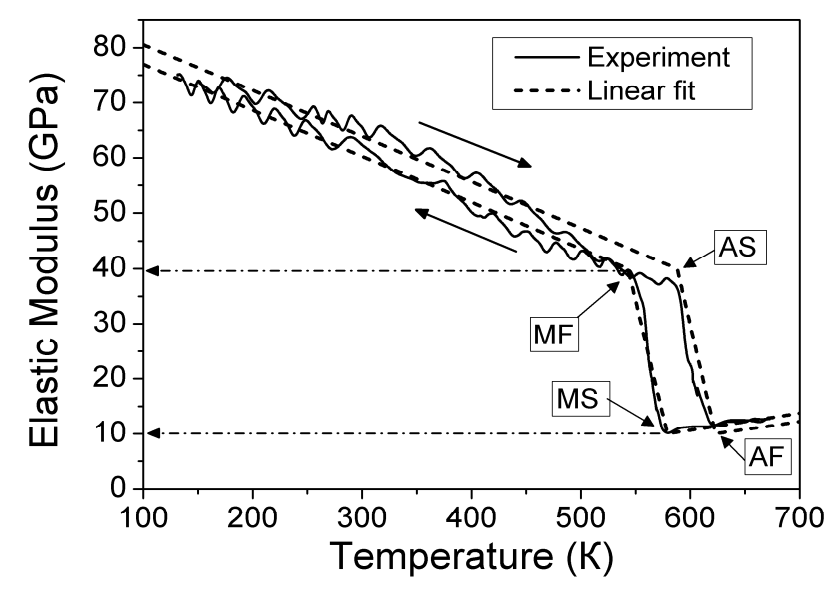

After measurements of the elastic modulus, Sample 1 was subjected to the stress-strain cycles for obtaining a stress-temperature phase diagram. The cycles were performed at different temperatures $T_{n}$, where $n=1,2 \ldots 15$ is the cycle number; the sample was heated to $673 \mathrm{~K}$ and held at this temperature during $10 \mathrm{~min}$ before each cycle. The critical stress values $\sigma_{A F}(T)$ and $\sigma_{M S}(T)$, which correspond to the finish of reverse MT and the start of forward MT, were determined from the stress-strain curves. These values are shown in Figure 7.

Figure 7. (a) Experimental stress values corresponding to the finish of reverse MT of Sample 1. (b) Experimental stress values corresponding to the start of forward MT of Sample 1 .

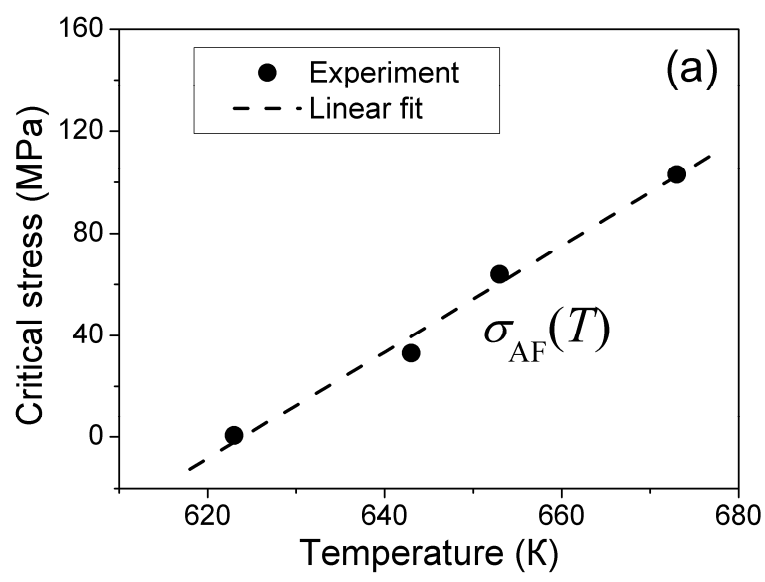


Figure 7. Cont.

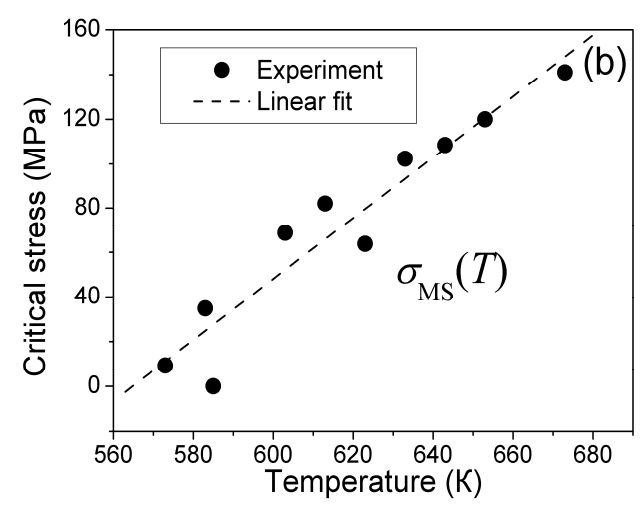

The temperature dependence of elastic modulus was measured again after the 15 th cycle to study the influence of thermomechanical cycling on the alloy properties. A drastic decrease of elastic modulus after the thermomechanical cycling was observed (see Figure 8).

Figure 8. (a) An influence of the thermomechanical cycling on the elastic modulus measured on cooling of Sample 1. (b) The influence of thermomechanical cycling on the elastic modulus measured on heating of Sample 1. The dashed lines show the theoretical temperature dependencies of elastic modulus that are derived in Section 5.4.
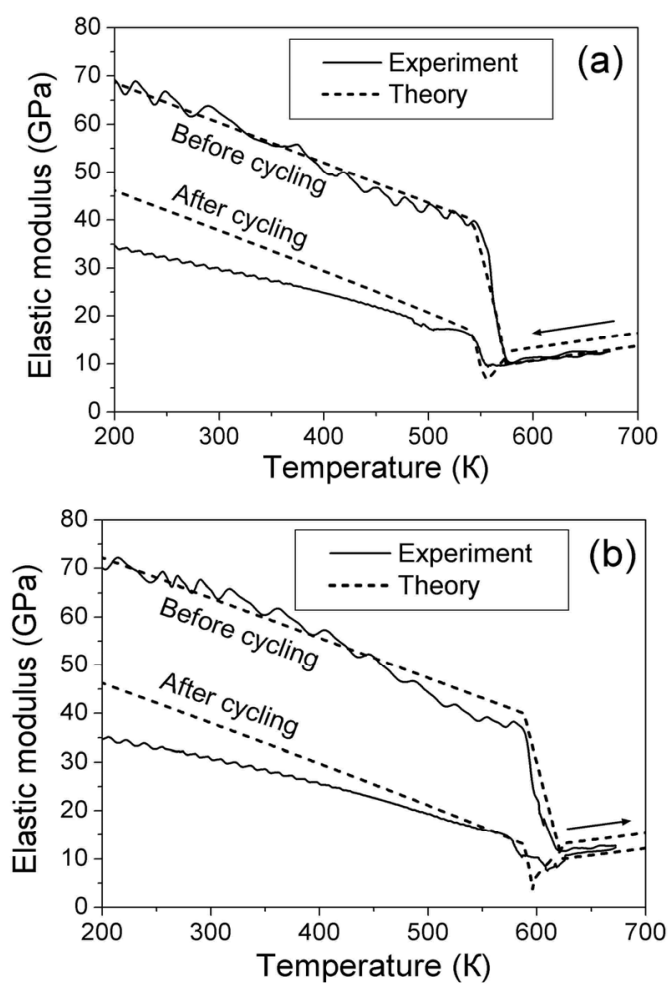

Sample 2 was used to investigate the influence of the cyclic stress-induced MTs on the superelastic properties. Ten sequential stress-strain cycles have been performed at constant temperature $673 \mathrm{~K}$. The stress-strain loops obtained during the first and tenth cycles are shown in Figure 9. 
Figure 9. The influence of mechanical cycling on the superelastic behavior of Sample 2 of $\mathrm{Ni}_{57.5} \mathrm{Mn}_{22.5} \mathrm{Ga}_{20.0}$ single crystal.

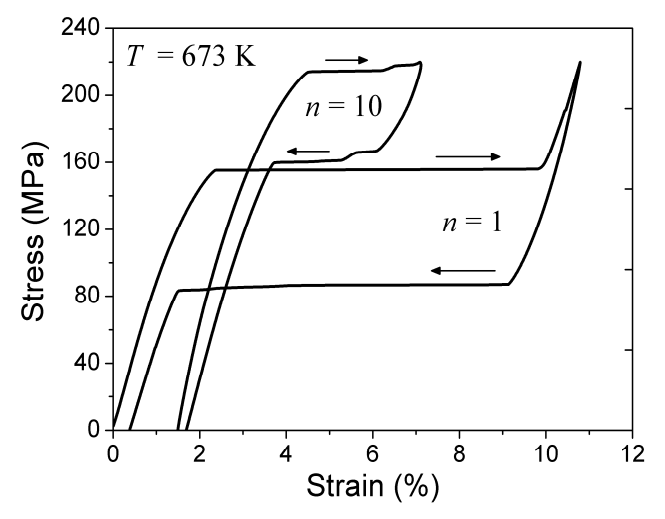

After mechanical cycling, the unloaded Sample 2 was subjected to 10 heating-cooling runs through MT and a noticeable shift of the reverse MT temperature $T_{R} \equiv\left(T_{A S}+T_{A F}\right) / 2$ was observed (see Figure 10). The shift of start of the forward MT temperature $T_{M S}$ proved to be comparable with the scatter of experimental points.

Figure 10. The influence of thermal cycling on the forward (bottom curve) and reverse (upper curve) MT temperatures of Sample 2.

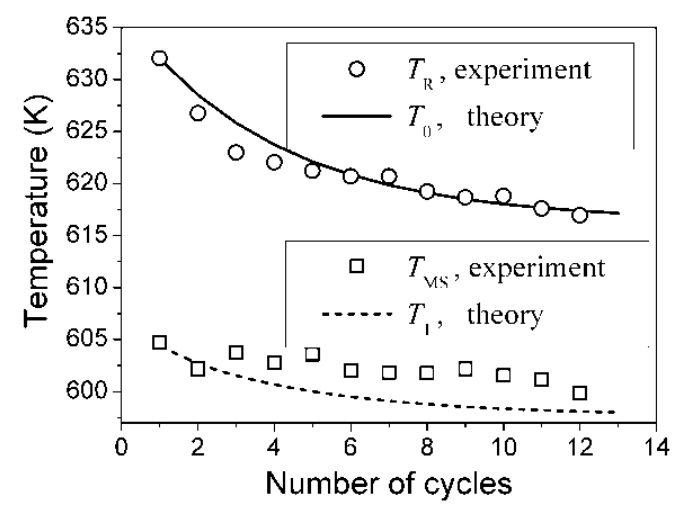

\subsection{Qualitative Explanation of Experimental Results}

The experiments demonstrate a noticeable softening of the elastic modulus of martensite after thermomechanical cycling (Figure 6). This effect suggests that cycling through the forward and reverse MTs destabilizes the martensitic state of the alloy. The suggestion is confirmed by an elevation of the plateau-like segments of the superelastic stress-strain loops in the course of the cyclic stress-induced MTs of Sample 2 (Figure 9): a destabilization of martensitic phase has to be accompanied by a decrease of MT temperature, and so, the martensite destabilization broadens an interval between the MT temperature and the constant temperature of alloy maintained during the cyclic mechanical loading. The movement of MT temperature away from the alloy temperature retards the stress-induced MT and elevates the critical stress value, which starts this MT [73-77].

The observed destabilization of martensitic phase admits a simple explanation. If the SMA is in the martensitic state during rather long time, the internal stress brings the real crystal lattice, which 
consists of the atoms situated in the regular crystallographic positions and crystal defects, into its equilibrium state, and therefore, stabilizes the martensitic phase. The cyclic MTs disturb an equilibrium state of the real crystal lattice and destabilize the martensitic phase. So, the martensite destabilization by cyclic MTs and martensite stabilization by aging can be considered as the opposite physical effects.

It is convenient to describe the martensite destabilization in terms of destabilizing internal stress, i.e., to count up the internal stress from its value reached before a thermomechanical cycling. In this case the destabilizing stress caused by cycling and the stabilizing stress that arises during the martensite aging are of the same order of magnitude with the opposite signs. Due to this, the expected absolute value of destabilizing stress can be roughly estimated from the experiments on martensite aging.

The martensite destabilization may be, in principle, caused by both isotropic and axial part of the internal stress, Equation 44. However, the absolute value of axial internal stress (about few tens of megapascals [63]) observed in the experiments on martensite aging is not sufficiently large to be responsible for the strong influence of cycling on the elastic modulus. In the same time, the elastic modulus can be changed by the internal pressure (see Sections 3 and 4). This assumption is supported by the comparatively large volume changes (about $0.5 \%$ [78]) observed during MTs of Ni-Mn-Ga alloys into a non-modulated tetragonal phase with $c / a>1$.

\subsection{Quantitative Description of the Martensite Destabilization Effects}

Physical considerations, which are essentially similar to those presented in Section 4.2, lead to the following dependence of destabilizing internal pressure on the cycle number $n$ :

$$
P^{(i)}(n)=P^{(i)}(\infty)\left[1-\exp \left(-n / n_{0}\right)\right],
$$

where $n_{0}$ is the characteristic integer prescribed by a rate of the defect subsystem reconfiguration, $P^{(i)}(\infty)=$ const (for more details see [37]). For the Ni-Mn-Ga alloys, the volume effect of MT is negative $\left(v_{M T}<0\right)$ and therefore, the destabilizing internal pressure must be negative, that is the cycling must increase the volume of the crystal.

The transformational properties of Ni-Mn-Ga alloys are described by minimization of the Gibbs potential, Equation 11. The Gibbs potential coefficients depend on the cycle number as

$$
\begin{aligned}
& c_{2}^{*}(T, n)=c_{2}(T)-3 a_{2} P^{(i)}(n) / c_{1}, \\
& a_{4}^{*}(n)=a_{4}-9 \Omega a_{2} P^{(i)}(n) / 2 c_{1}, \\
& b_{4}^{*}=b_{4}-a_{2}^{2} / 2 c_{1} .
\end{aligned}
$$

These dependencies were obtained from Equation 12 by substitution $P \rightarrow P^{(i)}(n)$.

The further analysis of problem is similar to that presented in the Sections 2 and 3. The austenitic phase is stable if $c_{2}^{*}(T, n)>0$, whereas the martensitic phase is stable for $c_{2}^{*}(T, n)<c_{t}^{*}(n)$. The equations $c_{2}^{*}\left(T_{1}, n\right)=c_{t}^{*}(n)$ and $c_{2}^{*}\left(T_{2}, n\right)=0$ prescribe the values of the lability temperatures $T_{1}(n)$ and $T_{2}(n)$. The shear elastic modulus of austenitic phase $C^{(A)} \equiv\left[C_{11}^{(A)}-C_{12}^{(A)}\right] / 2$ is related to the coefficient of the second-order energy term as

$$
C^{(A)}(T, n)=3 c_{2}^{*}(T, n) .
$$


The value of this modulus in the martensitic phase $C^{(M)}$ can be expressed through the equilibrium value of the order parameter $u_{0}$ as explained in the Section 3.2, the resultant expression

$$
C^{(M)}(T, n)=3 a_{4}^{*}(n) u_{0}^{*}(T, n)+6 b_{4}\left[u_{0}^{*}(T, n)\right]^{2}
$$

is similar to Equation 40 obtained there for the rhombohedral martensitic phase. The condition

$$
C^{(M)}\left(T_{M F}, n\right)=4 C^{(A)}\left(T_{M S}, n\right) .
$$

proves to be valid. (This condition is similar to Equation 42 and was derived in the same way). The experimental data presented in Figure 6 confirm the validity of this condition.

Now, let us use the experimental temperature dependence of the elastic modulus, measured before cycling of the Ni-Mn-Ga alloy (Figure 6), for a consistent description of the effect of cycling on this modulus (Figure 8) and MT temperatures (Figure 10). To this end, the Gibbs potential coefficients must be evaluated. It can be done assuming that the measured elastic modulus is close in value to the Young's modulus $E(T)$. The latter can be approximately expressed as $E^{(A, M)}(T) \approx 3 C^{(A, M)}(T)$ [49], because the soft elastic modulus of SMA is substantially smaller than its bulk modulus.

In view that the Landau theory does not describe the hysteresis phenomena properly, Equation 15 are considered to be applicable to both the forward and reverse MT. Therefore, the conditions $u_{0}\left(T_{M S}\right)=u_{0}\left(T_{A F}\right)$ and $u_{0}\left(T_{M F}\right)=u_{0}\left(T_{A S}\right)$ are accepted. These conditions together with Equation 52 result in the relationships $C^{(A)}\left(T_{M S}\right)=C^{(A)}\left(T_{A F}\right)$ and $C^{(M)}\left(T_{M F}\right)=C^{(M)}\left(T_{A S}\right)$. The experimental temperature dependencies of the elastic modulus validate these theoretical relationships (see Figure 6).

The coefficients $c_{2}(T), a_{4}$ and $b_{4}^{*}$ correspond to the state of the real crystal before cycling (that is to zero value of destabilizing stress). To evaluate these coefficients, the experimental dependencies of elastic modulus were approximated by the straight dashed lines shown in Figure 6 and Equations 18 and 19 with $C^{\prime}\left(T_{1,2}\right) \approx E\left(T_{M S, M F}\right) / 3$ were used. The approximation was made taking into account Equation 53. In addition, the experimental values of tetragonality ratio $c / a=1.22$ and the volume change $v_{M T} \approx-0.5 \%$ [78] were used for computations. These experimental values give rise to the estimations $u_{0}\left(T_{M F}\right)=2(c / a-1)=0.44, a_{4}=-10.1 \mathrm{GPa}, b_{4}^{*}=22.96 \mathrm{GPa}$ and $a_{2} / c_{1}=0.017$, which are the same for both forward and reverse MTs. Note, that the procedure of obtaining these estimations is similar to that described in Sections 3.3 and 4.2. The difference between the forward and reverse MTs arises when the different experimental temperature dependencies of the elastic modulus (see Figure 6) and correspondent characteristic temperatures $\left(T_{1}=T_{M S}, T_{2}=T_{M F}\right.$ for the forward MT, and $T_{1}=T_{A F}$, $T_{2}=T_{A S}$ for the reverse MT) are substituted into Equations 51 and 52.

Using values obtained above, the functions $c_{2}^{*}(T, n=15)$ and $a_{4}^{*}(n=15)$ were calculated for theoretical description of the influence of thermomechanical cycling on the temperature dependence of elastic modulus of Sample 1. The expression for $a_{4}^{*}(n)$ involves a dimensionless parameter $\Omega$. According to argumentation presented in Section 2.2, the value $\Omega=2 u_{0}^{-1}\left(T_{2}\right)$ was accepted. For the sake of definiteness, the value $n_{0}=4$ was used for computations. Characteristic values of the internal pressure $P^{(i)}(\infty)=-6 \mathrm{GPa}$ (for the forward MT) and $P^{(i)}(\infty)=-7.5 \mathrm{GPa}$ (for the reverse MT) were adjusted to achieve a reasonable agreement between the theoretical and experimental temperature dependencies of the elastic modulus. Figure 8 shows that an agreement between the experimental and 
theoretical dependencies takes place in rather wide temperature interval, $\sim 100 \mathrm{~K}$, below the martensite finish (for the forward MT) and austenite start (for the reverse MT) temperatures.

Both experiment and theory shows a significant reduction of the elastic modulus in the martensitic phase and a noticeable decrease of MT temperatures after cycling. These two features reveal a martensite destabilization effect.

Finally, the experimentally observed effect of thermal cycling on the reverse MT temperature (Figure 10) should be described theoretically. For this purpose, the experimentally determined temperature values $T_{R}(n)=\left[T_{A S}(n)+T_{A F}(n)\right] / 2$ are compared with the calculated ones $T_{0}(n)=\left[T_{1}(n)-T_{2}(n)\right] / 2$ (see Figure 10). The theory demonstrates a noticeable decrease of the $T_{0}$ temperature in the course of thermal cycling. The agreement between the theoretical and experimental data takes place if the value of internal pressure is put equal to $-1.5 \mathrm{GPa}$. The decrease of $T_{R}$ temperature demonstrates that the thermal cycling destabilizes the martensitic phase and the destabilizing pressure is close in value to the pressure that arises in the course of the mechanical cycling (see [37]). The theoretical dependence $T_{1}(n)$, which must be compared with the experimental $T_{M S}(n)$ values, is less pronounced. The experiment does not show a noticeable change of $T_{M S}$ temperature in view of the scattering of experimental temperature values, this scattering being comparable in magnitude to the temperature change predicted by theory.

\subsection{Summary of Destabilization Effects}

It can be summarized now that the destabilization of the $\mathrm{Ni}_{57.5} \mathrm{Mn}_{22.5} \mathrm{Ga}_{20.0}$ martensite has been discovered in the course of the experimental and theoretical studies of the cyclic MTs. The thermal and/or mechanical cycling of the shape memory alloy disturbs the equilibrium between the crystal lattice and defects. The deviation from the equilibrium is characterized by the internal stress, which destabilizes the martensitic phase. Due to the rather large volume effect of MT ( $\left.v_{M T} \approx-0.5 \%\right)$, the majority of experimentally effects of cycling observed in $\mathrm{Ni}_{57.5} \mathrm{Mn}_{22.5} \mathrm{Ga}_{20.0}$ alloy can be attributed mainly to the influence of negative internal pressure that is the isotropic part of internal stress. Both experiment and theory shows that the cyclic MTs of the alloy result in

(i) a decrease of the elastic modulus of martensitic phase (Figure 8);

(ii) a decrease of the MT temperature (Figure 10).

Experiment shows that the cycling increases noticeable the stress value, which in turn triggers the stress-induced MT. As it was shown in [37], this effect can be attributed to the influence of internal pressure as large as $-1.7 \mathrm{GPa}$, but a theoretical description of the stress-strain dependence is obviously impossible as long as the axial stress is disregarded. A consideration of the axial stress is beyond the scope of the present article.

The theoretically estimated negative internal pressure (causing an isotropic dilatation of the crystal lattice) is about $-2 \mathrm{GPa}$, in the case of mechanical or thermal cycling [37], and -6 GPa, in the case of thermomechanical cycling. A drastic difference between two estimated values of the internal pressure shows that combined thermomechanical cycling destabilizes the martensitic phase stronger than the pure mechanical or thermal cycling procedures performed in the experiments [37]. 


\section{Quasi-Second-Order Martensitic Transformations}

\subsection{General Considerations}

The numerous experiments show that MTs normally are the first-order phase transitions, but in some cases the quasi-second-order MTs are observed as well [48]. As far as the transformational behavior of SMAs is believed to be controlled by a principle of the minimum elastic energy, an observation of the second-order MT is in contradiction to a Lifshitz criterion (see [41] and references therein). The latter states that the second-order phase transition is impossible if the symmetry of the crystal allows the presence of cubic term of the order parameter in the Landau expansion for the free energy. It should be remembered that all quadratic, cubic and fourth-order invariant combinations of the order parameter components must be included in the expression for the free energy. This statement cannot be referred to as the "model assumption"; actually it is the basic principle of the theory of various physical phenomena in crystals (optical, ultrasonic, piezoelectric, ferroelectric, magnetic, and so on).

The Landau expansion for the free energy, Equation 4, involves the terms $a_{4} u_{3}\left(u_{3}^{2}-3 u_{2}^{2}\right)$ and $a_{5} u_{4} u_{5} u_{6}$, which are proportional to the third power of the order parameters of the cubic-tetragonal and cubic-rhombohedral MTs. It means that the martensitic transformations must be the first-order phase transition and the jumps of the values of lattice parameters and elastic modules at MT temperature must be predetermined by $a_{4}$ or $a_{5}$ values (for the alloys exhibiting cubic-tetragonal or cubic-rhombohedral MT, respectively).

An obvious disagreement between the theory of phase transitions and experimental observations of quasi-second-order MTs can be resolved in two ways.

First, the axial internal stress can be assumed to be present in the alloy specimens. This stress can reduce the cubic symmetry of crystal to tetragonal, rhombohedral, monoclinic or triclinic ones. In this case the MT is not accompanied by the symmetry change and appearance/disappearance of some physical value at MT temperature [79]. As so, the MT can be registered only by the observation of the jumps of the lattice parameters and jump-like changes of relevant physical values. If the axial stress exceeds some critical value, the jumps of lattice parameters vanish and the phase transition disappears; instead, the smooth nonlinear temperature dependence of lattice parameters takes place (for more details see $[80,81])$.

Second, the internal pressure can be induced by the crystal defects or chemical disorder. These factors are formally described by a renormalization of the energy coefficients $a_{4} \rightarrow a_{4}^{*}\left(P^{(i)}\right)$ and $a_{5} \rightarrow a_{5}^{*}\left(P^{(i)}\right)$, see Equations 45 and 50, respectively. Using the Equations 20 and 33, the renormalized coefficients can be expressed through the volume effect of MT and MT strain as follows:

$$
\begin{gathered}
a_{4}^{*}=a_{4}+\frac{v_{M T} P^{(i)}}{3 \varepsilon_{M}^{2}\left(T_{2}, 0\right)} \cdot \frac{\Omega}{\left[1+3 \Omega \varepsilon_{M}\left(T_{2}, 0\right)\right]}, \\
a_{5}^{*}=a_{5}+\frac{v_{M T} P^{(i)}}{3 \varepsilon_{[11]}^{2}\left(T_{2}, 0\right)} \cdot \frac{8 \Lambda}{\left[1+\Lambda \varepsilon_{[111]}\left(T_{2}, 0\right) / 3\right]} .
\end{gathered}
$$

Let some internal process or factor induce an internal pressure $P^{(i)}$. The positive/negative internal pressure decreases/increases the volume of an alloy. The negative/positive value $v_{M T}$ corresponds to 
the spontaneous decrease/increase of the volume during the forward MT. The internal process or factor results in the stabilization of martensitic phase if the spontaneous and forced volume changes are sign-like (see e.g., [25]), that is if the values $P^{(i)}$ and $v_{M T}$ are of the opposite signs. It means $P^{(i)} v_{M T}<0$ for the stabilizing pressure and $P^{(i)} v_{M T}>0$ for the destabilizing one.

The martensite stabilization results in an increase of the absolute value of MT strain, and therefore, denotes an increase of the absolute values of the energy parameters $a_{4}^{*}$ or $a_{5}^{*}$. If so, the values $a_{4}$ and $\Omega$ or $a_{5}$ and $\Lambda$ must be of the opposite signs. In the same time, the signs of parameters $a_{4}^{*}$ and $a_{5}^{*}$ are opposite to the signs of MT strains (see Equations 15 and 28). Therefore, the parameter $\Omega$ or $\Lambda$ must be of the same sign as the strain arising in the course of cubic-tetragonal or cubic-rhombohedral MT. The martensite stabilization accompanies the martensite aging. Therefore, the martensite aging denotes an increase of $\left|a_{4}^{*}\right|$ or $\left|a_{5}^{*}\right|$ magnitude and makes the first-order character of MT more pronounced.

It should be expected that the process or factor that destabilizes martensitic phase (reduces the MT temperature) denotes the decrease of $\left|a_{4}^{*}\right|$ or $\left|a_{5}^{*}\right|$ magnitudes and glosses over the first-order character of MT. The destabilizing internal pressure is of the same sign as the spontaneous volume change $v_{M T}$. It may be possible to observe a quasi-second order MT in presence of destabilizing internal pressure, which considerably reduces the absolute values of the coefficients $\left|a_{4}^{*}\right|$ and $\left|a_{5}^{*}\right|$.

\subsection{Application to the Ni-Mn-Ga and Au-Cd Alloys}

The energy coefficient $a_{4}^{*}$ has been evaluated for Ni-Mn-Ga alloy with $c / a<1\left(u_{0} \approx-0.12\right)$ in [41]. The reported in this work data show that the destabilizing internal pressure of about of $-3 \mathrm{GPa}$ reduces the $a_{4}^{*}$ value by one order of magnitude if the values $\Omega=2 u_{0}^{-1}$ and $v_{M T}=-0.5 \%$ are accepted for estimations. The choice of $\Omega$ value is explained in Section 2.3. The values of volume changes during MTs in the Ni-Mn-Ga alloys with $c / a<1$ are disputed: the X-ray diffraction data result in the large value $v_{M T} \approx-2 \cdot 10^{-2}$, while the values estimated from thermodynamic measurements are smaller by almost one order of magnitude (see [28] and references therein). In view of this ambiguity, the ratio $a_{4}^{*}(P) / a_{4}$ is presented in Figure 11 for the low $(0.1 \%)$, intermediate $(0.5 \%)$ and high $(2 \%)$ volume changes.

Figure 11. The influence of internal pressure on the coefficient of the third-order energy term, which predetermines the first-order character of martensitic transformation in the Ni-Mn-Ga alloy considered in [41].

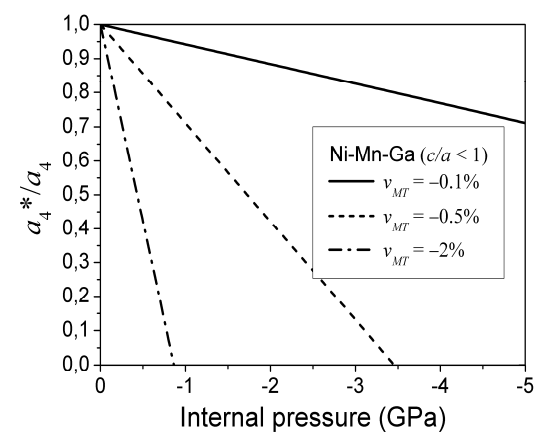


In the case of intermediate and large volume changes, the internal pressure can reduce the $a_{4}^{*}$ value by one order of magnitude. In this case the MT in the alloy considered in [41] may bear resemblance to the second-order phase transition. It should be noticed that the theoretical analysis of aging effects in Ni-Mn-Ga martensite with $c / a<1$ gave a comparatively low value of stabilizing pressure $P^{(i)}(t)<1 \mathrm{GPa}$ [35], but larger in the absolute value destabilizing pressure can be induced by the thermomechanical cycling of alloy specimens or some other mode of treatment.

The influence of internal pressure on the transformational and elastic properties of the rhombohedral Au-Cd martensite and tetragonal Ni-Mn-Ga martensite with $c / a>1$ was considered in the Sections 4 and 5 and the values of physical parameters inherent to these alloys were listed. It is worth to compute the energy coefficients, Equation 54, for these martensites. The results of computations are shown in Figure 12.

Figure 12. Influence of internal pressure on the coefficient of the third-order energy term, which predetermines the first-order character of martensitic transformation in the rhombohedral Au-Cd martensite and tetragonal Ni-Mn-Ga martensite, considered in $[36,37]$.

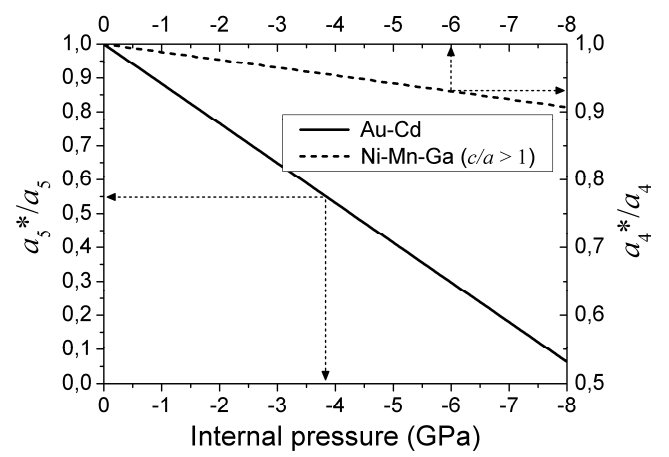

The stabilizing pressure $P^{(i)}=3.8 \mathrm{GPa}$ was estimated for the aged Au-Cd alloy in Section 4.2. The arrows in Figure 12 illustrate that the destabilizing pressure of the same absolute value reduces $a_{5}^{*}$ coefficient by factor of 0.55 .

The destabilizing pressure of about $-6 \mathrm{GPa}$ was induced by thermomechanical cycling of Ni-Mn-Ga martensite with $c / a>1$ (see [37] and Section 5.3). The arrows in Figure 12 illustrate that this pressure reduces $a_{4}^{*}$ value by factor of 0.93 only. The weak renormalization of the $a_{4}^{*}$ coefficient follows from the large value $\varepsilon_{M} \approx 15 \%$ being inherent to the considered Ni-Mn-Ga martensites: according to Equation 54, the renormalization of $a_{4}^{*}$ is proportional to the ratio of the dimensionless values $\Omega / \varepsilon_{M}^{2} \sim 1 / \varepsilon_{M}^{3}$, which is approximately equal to 300 for the Ni-Mn-Ga alloys with $c / a>1$ and is of the order of $-10^{4}$ for the Ni-Mn-Ga alloys with $c / a<1$.

It should be stressed that in view of an ambiguity in $\Omega, \Lambda$ and $P^{(i)}$ values, the data presented in Figures 11 and 12 have the illustrative character. Nevertheless, these data and the results reported in $[41,81]$ provide a plausible explanation of the quasi-second-order MTs in some shape memory alloys. Moreover, the consideration presented above shows that the resemblance between MT and the second-order phase transition presumes not only the certain alloy composition but also a special route of preparation of the alloy specimen. 


\section{Volume Changes Contributing to the Entropy in Magnetic Shape Memory Alloys}

\subsection{Elastic and Magnetic Components of the Entropy Change during MT}

Ferromagnetic and metamagnetic shape memory alloys (FSMAs and MetaSMAs) are attracting considerable interest due to the unique properties they show as a consequence of the coupling between their transformational properties and magnetism. The recent measurements of the entropy change $\Delta S$ accompanying the MTs in the different representatives of MetaSMAs, such as Ni-Mn-In and Ni-Mn-In-Co alloy systems, discovered one of the most obvious manifestations of this coupling. It has been found that $\Delta S$ strongly decreases with the distance between the MT temperature, $T_{M}$, and the Curie temperature, $T_{C} \geq T_{M}$ [31,32]. A similar decrease was observed when the available experimental data about the entropy change and transformation heat were collected and analyzed for the Ni-Mn-Ga FSMAs with $T_{C} \geq T_{M}$ [33]. It suggests that the magnetic subsystem of the FSMAs and MetaSMAs considerably contributes to the entropy change. Inasmuch as the spontaneous deformation of crystal lattice is considered as the key feature of MT, a coupling between the strain tensor components and magnetic moments of atoms, commonly referred to as magnetoelastic coupling [79], would be thereafter taken into account to estimate the magnetic contribution to the entropy change that accompanies MT. In the case of ferromagnetic ordering, the magnetoelastic coupling is described by the energy terms that are linear in the strain tensor and quadratic in the magnetization vector components. According to arguments advanced in the Sections 2.1 and 2.4, the magnetoelastic energy can be expressed through the magnetically induced internal stress, which, in its turn, can be subdivided into the isotropic and anisotropic parts. The isotropic part (magnetoelastic pressure) is responsible for volume magnetostriction.

In the absence of external forces, the Gibbs potential of thermodynamic system coincides with the Helmholtz free energy. The expression for the free energy of FSMA that exhibits cubic-tetragonal MT can be presented in the form

$$
F=F_{e l}\left(u_{2}, u_{3}\right)+F_{m}\left(M_{k}^{2}\right)+F_{m e}\left(M_{k}^{2}, u_{2}, u_{3}\right) .
$$

Elastic part of the free energy $F_{e l}$ is expressed through the linear combinations $u_{2}, u_{3}$ of diagonal strain tensor components by Equation 11 with $P=\sigma_{2}=\sigma_{3}=0$ :

$$
F_{e l}=\frac{1}{2} c_{2}(T)\left(u_{2}^{2}+u_{3}^{2}\right)+\frac{1}{3} a_{4} u_{3}\left(u_{3}^{2}-3 u_{2}^{2}\right)+\frac{1}{4} b_{4}^{*}\left(u_{2}^{2}+u_{3}^{2}\right)^{2} .
$$

The magnetic part of free energy $F_{m}\left(M_{k}^{2}\right)$ is the sum of spin-exchange and magnetic anisotropy energies expressed through the magnetization vector components $M_{k}$ as

$$
F_{m}=\frac{1}{2} J(T) \mathbf{M}^{2}+F_{A}\left(M_{k}^{2}\right)
$$

where $J(T)=\zeta\left(T-T_{C}\right) / T_{C}$ is the spin-exchange parameter, $T_{C}$ is the Curie temperature, and $\zeta$ is a dimensionless phenomenological constant. If the Curie temperature is near to room temperature, the spin-exchange energy is much greater than magnetic anisotropy energy $F_{A}\left(M_{k}^{2}\right)$. 
In accordance with the general principles formulated in Section 2.1 the expression for magnetoelastic energy is invariant with respect to the symmetry group of the cubic (paramagnetic austenitic) phase of the alloy. This expression is

$$
F_{m e}=-\delta_{e x} M^{2} u_{1}-\delta\left[\sqrt{3}\left(M_{x}^{2}-M_{y}^{2}\right) u_{2}+\left(2 M_{z}^{2}-M_{y}^{2}-M_{x}^{2}\right) u_{3}\right],
$$

where $\delta_{e x}$ and $\delta$ are the dimensionless magnetoelastic constants, describing the volume and axial magnetostriction of the single crystalline alloy, respectively [82]. The volume magnetostriction and axial magnetostriction arise because the spin-exchange energy and magnetic anisotropy energy of alloy vary in the course of deformation process.

The Equation 58 can be presented in the form

$$
F_{m e}=3 P^{(m e)} u_{1}-\frac{1}{6}\left(\sigma_{2}^{(m e)} u_{2}+\sigma_{3}^{(m e)} u_{3}\right)
$$

where

$$
P^{(m e)}=-\delta_{e x} M^{2} / 3
$$

is magnetoelastic pressure and the values

$$
\sigma_{2}^{(m e)}=6 \delta \sqrt{3}\left(M_{x}^{2}-M_{y}^{2}\right), \sigma_{3}^{(m e)}=6 \delta\left(2 M_{z}^{2}-M_{y}^{2}-M_{x}^{2}\right),
$$

represent the anisotropic components of magnetoelastic stress.

It is commonly known that the axial magnetostress must be taken into account when the anisotropic properties of FSMAs or non-scalar physical values (as the axial magnetic-field-induced strain) are considered. However, the volume magnetostriction and magnetoelastic pressure are caused by the spin-exchange interaction, whereas the anisotropic part of magnetoelastic energy and anisotropic part of magnetoelastic stress are mainly related to the spin-orbit interaction. Therefore, the isotropic part of magnetoelastic interaction is much larger than the anisotropic part, and so, the latter can be omitted when the influence of magnetoelastic coupling on the martensitic transformation heat and the relevant entropy change are considered. Noteworthy, in the Ni-Mn-Ga alloys, which are the most studied FSMAs, the magnetoelastic pressure exceeds the anisotropic magnetoelastic stress by two orders of magnitude [41,82-85], at least, and the disregard of this stress is substantiated by the numerical calculations. The neglect of anisotropic magnetic and magnetoelastic energy terms with respect to isotropic ones results in the expression

$$
F=F_{e l}\left(u_{2}, u_{3}\right)+\frac{1}{2} J(T) \mathbf{M}^{2}+3 P^{(m e)} u_{1} .
$$

In view that we are interested mainly in the magnetic contribution to the entropy change, the FSMAs, exhibiting the martensitic transformations in ferromagnetic state will be considered below.

The procedure of calculation of the entropy change caused by the phase transition is described by L.D. Landau and E.M. Lifshitz [79]. The calculation has to be performed in two steps. At the first step, the partial derivative $S=-\partial F / \partial T$ of free energy has to be calculated considering that the order parameter is temperature-independent. At the second step, the equilibrium values of the order parameter that are inherent to the austenitic and martensitic phases have to be substituted into the 
obtained expression for the entropy and the difference of the resultant entropy values $\Delta S \equiv S_{A}-S_{M}$ has to be calculated.

According to Landau theory, the coefficients of third- and fourth-order terms of elastic energy (Equation 56) are temperature independent. By differentiating Equation 62, one obtains the expression

$$
S=\frac{1}{2}\left(u_{2}^{2}+u_{3}^{2}\right) \frac{\partial c_{2}}{d T}+\frac{1}{2} \frac{\partial}{\partial T}\left[J(T) M^{2}(T)\right]-2 \delta_{e x} u_{1} M(T) \frac{\partial M(T)}{\partial T} .
$$

The free energy, Equation 62, and entropy, Equation 63, are counted of the entropy of paramagnetic cubic phase. In the ferromagnetic austenitic phase the values of the order parameter components and the concomitant $u_{1}$ value are caused by the ordinary magnetostriction and are much smaller than MT strain and volume change during MT. As so, these values can be put equal to zero. Due to this, the entropy of austenitic phase is approximately equal to

$$
S_{A}=\frac{1}{2} \frac{\partial}{\partial T}\left[J(T) M^{2}(T)\right]
$$

whereas the entropy of martensitic phase is expressed as

$$
S=S_{A}+\frac{1}{2} u_{0}^{2}(T) \frac{\partial c_{2}}{d T}-2 \delta_{e x} u_{1}(T) M(T) \frac{\partial M(T)}{\partial T} .
$$

It should be remembered that the "magnetic energy", Equation 57, is the energy of interaction between the magnetic moments of atoms in the high-symmetry phase, in our case, in the undistorted crystal lattice. By definition, the magnetoelastic energy is the total change of magnetic energy caused by the deformation of cubic lattice. That is why the derivative $\partial\left[J(T) M^{2}(T)\right] / \partial T$ is substituted in Equation 65 by the value Equation 64, which is inherent to the cubic ferromagnetic phase.

Subtracting $S_{A}$ value from the right side of Equation 65, one can present the entropy change as the sum of elastic and magnetic parts

$$
\Delta S=\Delta S_{e l}+\Delta S_{m a g}
$$

where

$$
\Delta S_{e l}=\frac{1}{2} u_{0}^{2}\left(T_{M F}\right)\left(\frac{\partial c_{2}}{d T}\right)_{T=T_{M F}},
$$

and

$$
\Delta S_{m a g}=-\frac{2}{3} \delta_{e x} v_{M T} M\left(T_{M F}\right)\left(\frac{\partial M}{\partial T}\right)_{T=T_{M F}} .
$$

Equation 67 describes the elastic part of the entropy change through the temperature derivative of the second-order energy coefficient, which is not directly measurable. This coefficient is related to the shear elastic modulus of austenitic phase as $c_{2}=C^{\prime} / 3$, but the interrelation between $c_{2}$ and elastic modulus of martensitic phase is more complicated (see Equation 52). However, the $c_{2}(T)$ is a monotone function and it can be assumed that the slope of $c_{2}(T)$ curve does not vary noticeably in the temperature interval $T_{M F}<T<T_{M S}$. In this case the elastic part of the entropy change can be estimated from the relationship 


$$
\Delta S_{e l}=\frac{2}{3}\left(\frac{a-c}{a}\right)^{2}\left(\frac{\partial C^{\prime}}{\partial T}\right)_{T \rightarrow T_{M S}}
$$

where $a$ and $c$ are the lattice parameters of a tetragonal phase.

A contribution of magnetic subsystem of the alloy, Equation 68, proved to be directly proportional to: (i) the temperature derivative of magnetization value; (ii) the volume change during MT; and (iii) the magnetoelastic constant. These points are physically clear because: (i)' both $\Delta S_{\text {mag }}$ and $\partial M / \partial T$ characterizes the change of degree of magnetic ordering; (ii)' the volume change characterizes the transformation of crystal lattice in the isotropic approximation; and (iii)' the volume magnetostriction constant characterizes the interdependence between the volume of alloy specimen and the intensity of spin-exchange process, which provides the main contribution to the magnetic energy expressed by Equation 57.

The magnetoelastic constant is not directly measurable. Its value can be determined, in principle, from the measurements of a volume magnetostriction. A spontaneous volume magnetostriction $v^{(m e)}(T)$ accompanies magnetic ordering. Using condition $\partial F / \partial u_{1}=0$ and Equations 4 and 58, it can be expressed as

$$
v^{(m e)}(T)=\delta_{e x} M^{2}(T) /\left(C_{11}+2 C_{12}\right) .
$$

Therefore, the magnetic entropy change

$$
\Delta S_{m a g}=-\frac{2}{3} v_{M T} v^{(m e)}\left(T_{M F}\right)\left(C_{11}+2 C_{12}\right) M^{-1}\left(T_{M F}\right)\left(\frac{\partial M}{\partial T}\right)_{T=T_{M F}}
$$

proves to be proportional to the product of volume change during MT and spontaneous volume magnetostriction.

The evaluation of the magnetic and elastic parts of the entropy change may be hampered by the difficulties in determination of the volume magnetostriction and temperature derivatives from the experimental $M(T)$ and $C^{\prime}(T)$ values. In this regard, the relationship

$$
T_{M S}-T_{M F} \approx \frac{1}{\Delta S} \cdot \frac{3 \varepsilon_{M}^{2} C^{\prime}\left(T_{M S}\right)}{2 m}
$$

may be useful [33]. Moreover this expression is very significant in its own right because it implies a linear interdependence between the width of the temperature interval of MT and $\Delta S^{-1}$ value and so, can be verified easily in the experiment.

Another way of the determination of magnetic entropy change is proposed in [33] for the case when the experimental temperature dependence of magnetization of FSMA with $T_{M S}<T_{C}$ obeys the standard equations

$$
M(T)= \begin{cases}\tanh \left[\frac{T_{C} M(T)}{T M_{0}}\right], & \text { if } T>T_{M S}, \\ \tanh \left[\frac{T^{*} M(T)}{T M_{0}}\right], & \text { if } T<T_{M F},\end{cases}
$$

where $M_{0}=M(0)$, Curie temperature $T_{C}$ is the temperature of ferromagnetic ordering of FSMA in austenitic phase and $T^{*}$ is referred to as the "virtual" temperature of ferromagnetic ordering of the 
martensitic phase. The difference between the actual and virtual temperatures of ferromagnetic ordering can be related to volume change during MT: the $u_{1}$ variable is equal to zero before the forward MT and to the constant value $v_{M T} / 3$ immediately after it. According to Equations 60 and 62, the volume change results in the renormalization of the spin exchange parameter

$$
J(T) \rightarrow J^{*}(T)=J(T)-\frac{2}{3} \delta_{e x} v_{M T} .
$$

The temperatures $T_{C}$ and $T^{*}$ satisfy the conditions $J\left(T_{C}\right)=0$ and $J^{*}\left(T^{*}\right)=0$, which result in the relationship $T^{*}=T_{C}(1+\kappa / \zeta)$ involving the dimensionless parameters $\zeta$ and

$$
\kappa \equiv(2 / 3) \delta_{e x} v_{M T} .
$$

The parameter $\kappa$ relates the magnetic entropy change to the magnetization of FSMA (see Equation 68).

The experiments with different FSMAs demonstrate a stepwise change of magnetization value in the narrow temperature interval below $T_{M S}$. The characteristic temperatures $T_{C}$ and $T^{*}$ can be found by fitting the solutions of Equation 73 to the high-temperature $\left(T>T_{M S}\right)$ and low-temperature $\left(T>T_{M F}\right)$ segments of the experimental $M(T)$ curve. The relationship $\zeta \approx n k_{B} T_{C}$ ( $n$ is the amount of magnetic atoms in the unit volume) enables the estimation of the parameter

$$
\kappa=\zeta\left(T^{*}-T_{C}\right) / T_{C}
$$

and magnetic entropy change, Equation 68.

The findings of general theoretical analysis of the entropy change during MT can be summarized in the following principal points:

I) The entropy change during MT is inversely proportional to the width of the temperature interval of mixed (austenitic-martensitic) state.

II) The magnetoelastic energy of a deformable magnetic solid, such as FSMA, is the difference between the magnetic energies of its deformed and undeformed states.

III) The magnetic contribution to the total entropy change caused by the deformation of magnetic solid is the partial temperature derivative of magnetoelastic energy taken with the opposite sign.

IV) The ordinary magnetostriction of FSMA is substantially smaller than the MT strain $v_{M T}$, and the axial magnetostriction is much smaller than the spontaneous volume magnetostriction $v^{(m e)}$ in the case if the Curie temperature is of the order of room temperature. In this case the magnetic entropy change is directly proportional to the product $v_{M T} v^{(m e)}\left(T_{M F}\right)$.

V) Not only the magnetic entropy change during MT but also the magnetization jump is caused by the magnetoelastic coupling and interrelated with the product $v_{M T} v^{(m e)}\left(T_{M F}\right)$. This follows from the Equations 70,73,75 (for more details see [33,83]). 


\subsection{Magnetic Entropy Change during MT of Ni-Mn-Ga Alloy}

The Ni-Mn-Ga alloys undergoing the cubic-tetragonal MTs in the ferromagnetic state are the most studied to date FSMAs. The magnetization functions of some of these alloys are well described by the Equation 73, and therefore, the virtual Curie temperatures and $\kappa$ values can be estimated for these alloys by fitting theoretical $M(T)$ curves to the experimental values of magnetization [84]. In particular, the fitting procedure was performed for a $\mathrm{Ni}_{52.6} \mathrm{Mn}_{23.5} \mathrm{Ga}_{23.9}$ alloy (Alloy 1) that undergoes MT to the 5-layered tetragonal phase with $c / a<1$. For this alloy $T_{M F} \approx 275 \mathrm{~K}$, a good fit of the theoretical $M(T)$ curves to experimental values of the magnetization takes place for $T_{C}=375 \mathrm{~K}, T^{*}=405 \mathrm{~K}$ and $M_{0}=715 \mathrm{G}$ [84]. For the Ni-Mn-Ga alloy family, the estimation $\zeta M_{0}^{2} \approx 0.1 \mathrm{GPa}$ is valid [83], thus the Equation 76 gives the value $\kappa=150$. In this case the numeric computation of $M(T)$ function and its derivative results in the estimation $\Delta S_{\text {mag }}=14.3 \mathrm{~J} \mathrm{~kg}^{-1} \mathrm{~K}^{-1}$. This entropy change corresponds to the heat exchange $q_{m a g}=T_{0} \Delta S_{m a g}=4.07 \mathrm{~J} \mathrm{~g}^{-1}$. It is astonishing that the theoretical estimation of the "magnetic" heat exchange is very close to the experimental value of total transformation heat $q \approx 4.2 \mathrm{~J} \mathrm{~g}^{-1}$ reported for Alloy $1[78,86]$. The experimental value of transformation heat corresponds to the total entropy change of $14.7 \mathrm{~J} \mathrm{~kg}^{-1} \mathrm{~K}^{-1}$.

The solid line in Figure 13 shows the magnetic entropy change $\Delta S_{m a g}$ that was computed from Equation 68 by setting different $T=T_{M F}$ values in the argument of $M(T)$ function keeping fixed the rest of parameters such as those shown above for Alloy 1. The experimental values of the total entropy changes are shown by circles in Figure 13. To present these values, we collected the available experimental data for $\mathrm{Ni}-\mathrm{Mn}-\mathrm{Ga}$ martensites with $c / a<1$ [30,87-91]. The arrow points to the experimental value obtained for Alloy 1 . The theoretical dependence of the magnetic entropy change on the temperature difference $T_{C}-T_{M F}$ demonstrates that $\Delta S_{\text {mag }}$ quickly increases when the MT temperature approaches the Curie temperature and is less than $5 \mathrm{~J} \mathrm{~kg}^{-1} \mathrm{~K}^{-1}$ for the alloys with $T_{C}-T_{M F}>200 \mathrm{~K}$. The experimental values obviously correlate with the computed ones in spite of the fact that the different alloys are characterized by different $M(T)$ curves, while the curve used for computations is relevant only to the Alloy 1. The proximity of the experimental points to the theoretical curve suggests that the magnetic part of the total transformation heat exceeds all nonmagnetic parts for the Ni-Mn-Ga alloys with $T_{C}-T_{M F}<150 \mathrm{~K}$, at least. It should be noted, that the same conclusion can be deduced from the experimental data collected for different Ni-Mn-In and Ni-Mn-In-Ga alloys [92].

The calculated values of the magnetic entropy change are very close or even exceed the values of total entropy changes measured for Ni-Mn-Ga alloys. It suggests that the magnetic entropy change is overestimated. The overestimation can be caused, in particular, by the inaccuracy in the evaluation of parameter $\zeta$, which is absent in the equation for the magnetic entropy change and Equation 75 but is involved in Equation 76. It should be noticed, however, that a clear physical reason for the underestimation of the total entropy change exists. On the one hand, the cubic crystal lattice transforms to the spatially inhomogeneous martensitic state when the latent transformation heat is measured. The martensitic state is not completely self-accommodated and its energy is higher than the energy of thermodynamically equilibrium tetragonal phase considered above. Therefore, the measured 
transformation heat is smaller than the latent heat, which characterizes the transformation from the cubic to the equilibrium tetragonal phase.

Figure 13. Theoretical values of magnetic entropy change (line) and experimental values of total entropy change (circles) at MTs in the Ni-Mn-Ga alloys.

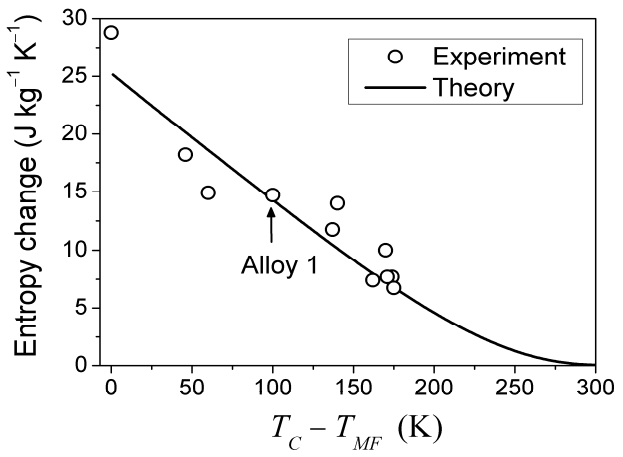

\subsection{Magnetic Entropy Change during MT of a Quasi-Stoichiometric Ni-Fe-Ga Alloy}

The temperature dependence of saturation magnetization of $\mathrm{Ni}_{53.5} \mathrm{Fe}_{19.5} \mathrm{Ga}_{27}$ alloy (Alloy 2) with $M_{0} \approx 480 \mathrm{G}, T_{C} \approx 330 \mathrm{~K}$ and $T_{M F} \approx 250 \mathrm{~K}$ is measured in [93]. This dependence is not described by Equation $73[33,83]$ and the virtual temperature of ferromagnetic ordering cannot be determined in view of an ambiguity in the extrapolation of experimental $M(T)$ dependence measured in the martensitic phase to the temperature range $T>T_{M F}$. However, the experimental temperature dependence of magnetization measured in the martensitic phase (that is for $T_{C}-T>80 \mathrm{~K}$ ) can be used to illustrate that the magnetic entropy change drastically depends on the shape of $M(T)$ curve. For this purpose the computations could be performed in two ways: (i) using the experimental $M(T)$ curve; and (ii) using the solution of Equation 73 with $M_{0}=480 \mathrm{G}$ and $T_{C}=330 \mathrm{~K}$. The $\kappa$ and $\zeta$ values estimated above for Alloy 1 can be accepted, because in this case Equation 73 gives the magnetization jump $M\left(T_{M F}\right)-M\left(T_{M S}\right) \approx 15 \mathrm{G}$, which follows also from the experimental $M(T)$ curve presented in $[33,83]$.

It should be emphasized that Equation 73 describes the electron gas in the Weiss molecular field, that corresponds to the widely used Bragg-Williams approximation (see, e.g., [93]). As it is seen from Figure 14, the Bragg-Williams approximation is not applicable to Alloy 2. Therefore, the magnetic entropy changes, which accompany MTs in Ni-Fe-Ga alloys, cannot be evaluated using this equation. Moreover, the virtual temperature of ferromagnetic ordering cannot be calculated and the parameter $\kappa$ relating $\Delta S_{m a g}$ to $M(T)$ dependence cannot be estimated from Equation 76. Therefore, this parameter has to be evaluated from Equation 75. In this case, the magnetoelastic constant must be determined and the volume change must be measured or estimated in some way.

The experimental values of lattice parameters reported for Alloy 2 [94] result in the estimation $v_{M T} \approx-3 \times 10^{-2}$, which corresponds to the value $u_{1} \approx-0.01$. It is noteworthy that in the case of Alloy 1 this value of the volume change gave rise to the above used value of $\kappa$ and provided for the excellent match between experimental and theoretical magnetization curves [84]. So, the used above value $\kappa=150$ may be correct for Ni-Fe-Ga alloys if the constant $\delta_{0} \equiv \delta_{e x} M^{2}\left(T_{0}\right)$ is approximately equal to 
$\delta_{0}=-0.4 \mathrm{GPa}$ [83], which was estimated before for Ni-Mn-Ga alloys. To the best of our knowledge, the magnetoelastic constants are still not evaluated for Ni-Fe-Ga alloys.

Figure 14. Theoretical values of magnetic entropy change computed using the experimental values of saturation magnetization (solid line) and the solution of Equation 73.

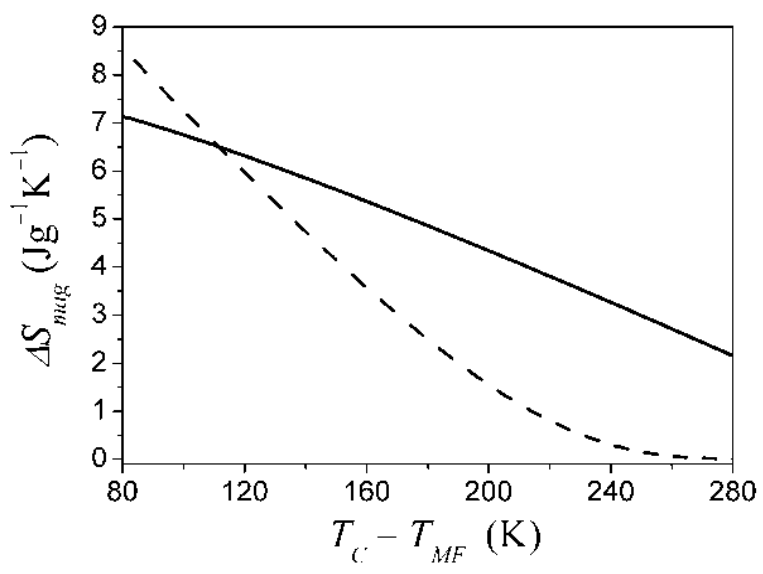

\subsection{Summary on the Entropy Change}

The consistent analysis of the entropy change that accompanies MT in FSMA demonstrates the important features of this physical value.

(i) The total entropy change during MT is inversely proportional to the width of the temperature interval of mixed two-phase state.

(ii) The elastic part of the entropy change is proportional to the value of temperature derivative of the shear elastic modulus. This feature illustrates that the cubic-tetragonal MT is caused by the instability of crystal lattice with respect to the vibrations corresponding to the $\mathrm{TA}_{2}$ [110] phonon mode and to the softening of this phonon mode which leads to a decrease of $C^{\prime}$ value in the vicinity of MT. In practice, the evaluation of $\Delta S_{e l}$ from the temperature dependencies of shear modules of SMAs is hampered by an uncertainty in the experimental values of these modules.

(iii) The elastic part of the entropy change is proportional to the squared tetragonal distortion of the unit cell, $(1-c / a)^{2}$, which is about of $4 \times 10^{-2}$ for the Ni-Mn-Ga alloys with $c / a>1$, $T_{M S}>T_{C}$ and $3 \times 10^{-3}$ for the alloys with $c / a<1, T_{M S}<T_{C}$. This feature is in line with the fact that the observed in [91] entropy change is noticeably larger for the Ni-Mn-Ga alloys with $T_{M S}>T_{C}$ than for those with $T_{M S}<T_{C}$.

(iv) In the case of FSMAs with $T_{M S}<T_{C}$, the Landau theory confirms a crucial role of the interaction between the magnetic and elastic subsystems in the formation of thermodynamic characteristics of the MT. The magnetic part of the entropy change estimated from $M(T)$ curves for Ni-Mn-Ga alloy (Alloy 1) appeared to be close in value to the experimentally observed total entropy change. In this connection, the entropy change demonstrates a sharp decrease when the MT temperature of the alloy moves away from the Curie temperature. The experiments show that this is a common feature of the Ni-Mn-Ga, Ni-Mn-In and 
Ni-Mn-In-Co alloy systems [32]. Recently, a pronounced dependence of $\Delta \mathrm{S}$ on $T_{C}-T_{M F}$ has been observed also in Ni-Mn-Ga-Co alloys [95].

(v) The computations illustrate that the evaluation of the magnetic entropy change from the temperature dependence of magnetization is very sensitive to the character of this dependence (see Figure 14). Moreover, it occurs that the standard equations corresponding to the Bragg-Williams approximation do not describe the magnetization of Ni-Fe-Ga alloys. It can be concluded, therefore, that the careful study of the magnetic structure and magnetization of every alloy system must be carried out for the correct evaluation of magnetic entropy change.

(vi) Equation 71 shows that the magnetic entropy change is proportional to the volume magnetostriction. Therefore, a careful theoretical analysis of the effect of spontaneous and forced magnetostriction on the characteristic MT temperatures will be very important.

The inverse proportionality of $\left(T_{M S}-T_{M F}\right)$ to $\Delta S$ was verified very recently in the experiments with Ni-Mn-In alloys (see Figure 15) [96].

Figure 15. The experimental verification of the inverse proportionality of two-phase temperature interval to entropy change [96]. The dashed line presents a linear fit to the experimental points.

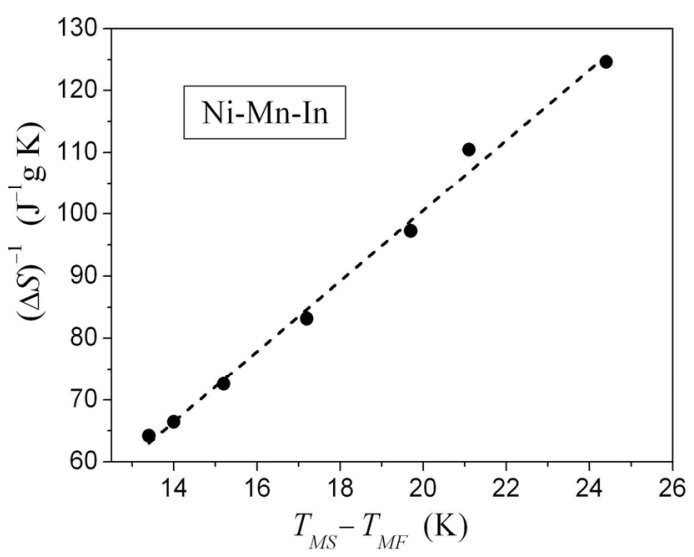

The following important remark about the value of volume change during MT is appropriate. The agreement between the theoretical and experimental values, illustrated by Figure 13, is achieved for $\kappa=150$. This value corresponds to the magnetoelastic constant $\delta_{e x} M_{0}^{2} \approx 0.4 \mathrm{GPa}[83,84]$ and volume change of the order of $1 \%$ directly given for Ni-Mn-Ga alloys by the $X$-ray data, while the Clausius-Clapeyron relationship results in a value for the volume change of about $0.1 \%$ estimated from the shift of MT temperature by the hydrostatic compression of Ni-Mn-Ga. The reduction of the volume change by one order of magnitude can be compensated only by the proportional increase of the magnetoelastic constant. The value $\delta_{e x} M_{0}^{2} \approx 2 \mathrm{GPa}$ was reported in [85].

\section{Discussion}

The volume conservation principle is often used for a comprehension of the thermoelastic behavior of shape memory alloys, while a few works include the consideration of equilibrium volumes of 
different phases. (The excellent first principle calculations of this kind are performed in [97]). In the same time, the symmetry conforming theory shows that the volume change is an indispensable feature of the cubic-tetragonal and cubic-rhombohedral martensitic transformations. It may be expected that this conclusion is also valid for the MTs to the orthorhombic and monoclinic phases because one of MT strain components often being much greater than others. The theory relates the volume change to MT strain via the certain combinations of the third- and fourth-order elastic modules, but the values of these combinations are not known at present. On the one hand, it shows that the appearance of small MT strain may be accompanied by the comparatively large volume change, and vice versa, the large MT strain may occur with very small volume change. On the other hand, an uncertainty in the used for computations ratios of the third- and fourth order modules (parameters $\Lambda$ and $\Omega$ ) retards the quantitative analysis of experimental results.

In spite of difficulties, the combined (experimental and theoretical) study of transformational properties of nonmagnetic and ferromagnetic shape memory alloys indicates the noticeable influence of the volume effect of MT and volume magnetostriction on the elastic and thermodynamic properties of these alloys.

The importance of the volume effect of MT can be explained in a simple manner. The volume change during MT results in the energy density change, which can be estimated by the order of magnitude as $\left|\Delta F_{1}\right| \sim\left|v_{M T}\right| B \sim 0.1-1 \mathrm{GPa}$, because the bulk elastic modulus $B$ is of the order of $100 \mathrm{GPa}$ and the inequalities $10^{-3} \leq v_{M T} \leq 10^{-2}$ are true in most cases. The spontaneous shear of crystal lattice during MT results in the energy density change $\left|\Delta F_{2}\right| \sim\left|\varepsilon_{M}\right| C^{\prime} \sim 0.1-1 \mathrm{GPa}$ because the MT strain satisfies the inequalities $10^{-2} \leq \varepsilon_{M} \leq 10^{-1}$ and the shear modulus of shape memory alloy is of the order of $10 \mathrm{GPa}$ in the vicinity of MT temperature. The energy changes $\Delta F_{1}$ and $\Delta F_{2}$ are in general of the same order of magnitude, and therefore, the volume effect of MT cannot be neglected a priory: the disregard of this effect must be substantiated in every case by the numerical evaluations.

We hope that the conclusion about the importance of volume changes will attract the attention of different researches teams and may be useful for planning the further experimental and theoretical studies of shape memory alloys.

\section{Acknowledgments}

V.A.L. is grateful to U. K. Rößler and M. E. Gruner for useful discussions during his stay in IFW, Dresden. A financial support from the Project No. MAT2011-28217-C02-01,02, by the Spanish Ministry of Science and Innovation, Project №0112U001009 by National Academy of Sciences of Ukraine (NASU) and STCU-NASU grant №5715 are greatly acknowledged.

\section{References}

1. Christian, J.W. Theory of Phase Transformations in Metals and Alloys; Pergamon Press: New York, NY, USA, 1975; pp. 1-973.

2. Khachaturyan, A.G. Theory of Structural Transformations in Solids; Wiley: New York, NY, USA, 1983; pp. 1-574.

3. Otsuka, K.; Wayman, C.M. Shape Memory Materials; Cambridge University Press: Cambridge, UK, 1998; pp. 1-267. 
4. Lagoudas, C. Shape Memory Alloys: Modeling and Engineering Applications; Springer-Verlag: New York, NY, USA, 2007; pp. 1-435.

5. Kohl, M. Shape Memory Microactuators; Springer-Verlag: Berlin, Germany, 2004; pp. 1-245.

6. Hannula, S.-P.; Söderberg, O.; Jämsä, T.; Lindroos, V.K. Shape memory alloys for biomedical applications. Adv. Sci. Technol. 2006, 49, 109-118.

7. Humbeeck, J.V. Non-Medical applications of shape memory alloys. Mater. Sci. Eng. 1999, 273-275, 134-148.

8. O’Handley, R.C.; Allen, S.M. Shape-Memory Alloys, Magnetically Activated Ferromagnetic Shape-Memory Materials. In Encyclopedia of Smart Materials; Schwartz M., Ed.; Wiley: New York, NY, USA, 2002; pp. 936-951.

9. Söderberg, O.; Ge, Y.; Sozinov, A.; Hannula, S.-P.; Lindroos, V.K. Giant Magnetostrictive Materials. In Handbook of Magnetic Materials; Buschow, J., Ed.; Elsevier Science: Amsterdam, the Netherlands, 2006; pp. 1-39.

10. Chernenko, V.A. Advances in Shape Memory Materials; TTP: Zurich, Switzerland, 2008; pp. 1-302.

11. Chernenko, V.A. Advances in Shape Memory Materials; TTP: Zurich, Switzerland, 2011; pp. 1-231.

12. Entel, P.; Buchelnikov, V.D.; Khovailo, V.V.; Zayak, A.T.; Adeagbo, W.A.; Gruner, M.E.; Herper, H.C.; Wassermann, E.F. Modelling the phase diagram of magnetic shape memory Heusler alloys. J. Phys. D Appl. Phys. 2006, 39, 865-889.

13. Acet, M.; Manosa, L.; Planes, A. Magnetic-Field-Induced Effects in Martensitic Heusler-Based Magnetic Shape Memory Alloys. In Handbook of Magnetic Materials; Buschow, J., Ed.; Elsevier Science: Amsterdam, the Netherlands, 2011; pp. 232-282.

14. Entel, P.; Siewert, M.; Gruner, M.E.; Herper, H.C.; Comtesse, D.; Arroyave, R.; Singh, N.; Talapatra, A.; Sokolovskiy, V.V.; Buchelnikov, V.D.; Albertini, F.; et al. Complex magnetic ordering as a driving mechanism of multifunctional properties of Heusler alloys from first principles. Eur. Phys. J. B 2013, 86, 65-75.

15. Murray, S.J.; Marioni, M.; Allen, S.M.; O’Handley, R.C.; Lograsso, T.A. 6\% magnetic-field-induced strain by twin-boundary motion in ferromagnetic Ni-Mn-Ga. Appl. Phys. Lett. 2000, 77, 886-888.

16. Heczko, O.; Sozinov, A.; Ullakko, K. Giant field-induced reversible strain in magnetic shape memory NiMnGa alloy. IEEE Trans. Magn. 2000, 36, 3266-3268.

17. Sozinov, A.; Lanska, N.; Soroka, A.; Zou, W. 12\% magnetic field-induced strain in Ni-Mn-Ga-based non-modulated martensite. Appl. Phys. Lett. 2013, 102, 021902:1-021902:5.

18. Warlimont, H.; Delaey, L. Martersitic. Transformations in Copper-, Silver- and Gold-Based Alloys; Pergamon Press: New York, NY, USA, 1974; pp. 1-379.

19. Kokorin, V.V.; Samsonov, Yu.I.; Khshanovskiy, L.F.; Chernenko, V.A.; Shevchenko, O.M.; Volume change during $\gamma-\alpha$ transformation in iron-nickel base alloys. Phys. Met. Metall. 1991, 71, 141-146.

20. Maki, T.; Kobayashi, K.; Minato, M.; Tamura, I. Thermoelastic martensite in an ausaged Fe-Ni-Ti-Co alloy. Scripta. Met. 1984, 18, 1105-1109. 
21. Maki, T.; Furutani, S.; Tamura, I. Shape memory effect related to thin plate martensite with large thermal hysteresis in ausaged Fe-Ni-Co-Ti alloy. ISIJ Int. 1989, 29, 438-445.

22. Kokorin, V.V.; Chernenko, V.A. Reversion stress evaluation in Fe-Ni-Co-Ti alloys. In Proceedings of the Second International Conference on Shape Memory and Superelastic Technologies, Pacific Grove, CA, USA, 1997; pp. 119-124.

23. Jost, N.; Escher, K.; Donner, P.; Sade, M.; Halter, K.; Hornbogen, E. Steels with shape memory. WIRE 1990, 40, 639-640.

24. Gefen, Y.; Halwany, A.; Rosen, M. Effect of hydrostatic pressure on the cubic-orthorhombic phase transformation in Au-47.5 at\% Cd alloy. Phil. Mag. 1973, 28, 1-9.

25. Kakeshita, T.; Yoshirnura, Y.; Shimizu, K.; Endo, S.; Akahama Y.; Fujita, F.E. Effect of hydrostatic pressure on martensitic transformations of $\mathrm{Cu}-\mathrm{Al}-\mathrm{Ni}$ shape memory alloy. Trans. Jpn. Inst. Metals 1988, 29, 781-789.

26. Kakeshita, T.; Shimizu, K. Effects of hydrostatic pressure on martensitic transformations. Mat. Trans. JIM 1997, 38, 668-681.

27. Zakrevskiy, I.G.; Kokorin, V.V.; Chernenko, V.A. Baroelastic martensitic transformation in Fe-Ni-Co-Ti alloy. Soviet Phys. Dokl. 1989, 34, 73-74.

28. Chernenko, V.A.; L'vov, V.A. Thermodynamics of martensitic transformations affected by hydrostatic pressure. Phil. Mag. A 1996, 73, 999-1008.

29. Aseguinolaza, I.R.; Reyes-Salazar, I.; Svalov, A.V.; Wilson, K.; Knowlton, W.B.; Mullner, P.; Barandiaran, J.M.; Villa, E.; Chernenko, V.A. Transformation volume strain in Ni-Mn-Ga thin films. Appl. Phys. Lett. 2012, 101, 241912:1-241912:4.

30. Khovailo, V.V.; Takagi, T.; Tani, J.; Levitin, R.Z.; Cherechukin, A.A.; Matsumoto, M.; Note, R. Magnetic properties of $\mathrm{Ni}_{2.18} \mathrm{Mn}_{0.82} \mathrm{Ga}$ Heusler alloys with a coupled magnetostructural transition. Phys. Rev. B 2002, 65, 092410:1-092410:4.

31. Ito, W.; Imano, Y.; Kainuma, R.; Sutou, Y.; Oikawa, K.; Ishida, K. Martensitic and magnetic transformation behaviors in Heusler-type NiMnIn and NiCoMnIn metamagnetic shape memory alloys. Metall. Mater. Trans. A 2007, 38, 759-766.

32. Kustov, S.; Corró, M.L.; Pons, J.; Cesari, E. Entropy change and effect of magnetic field on martensitic transformation in a metamagnetic Ni-Co-Mn-In shape memory alloy. Appl. Phys. Lett. 2009, 94, 191901:1-191901:3.

33. L'vov, V.A.; Cesari, E.; Recarte, V.; Pérez-Landazábal, J.I. Entropy change of martensitic transformation in ferromagnetic shape memory alloys. Acta Mater. 2013, 61, 1764-1772.

34. Xu, X.; Ito, W.; Umetsu, R.Y.; Koyama, K.; Kainuma, K.; Ishida, K. Kinetic arrest of martensitic transformation in Ni33:0Co13:4Mn39:7Ga13:9 metamagnetic shape memory alloy. Mater. Trans. 2010, 51, 469-471.

35. L'vov, V.A.; Kosogor, A.; Söderberg, O.; Hannula, S.-P. The symmetry-conforming theory of martensite aging. Mater. Sci. Forum 2010, 635, 13-19.

36. Kosogor, A.; L'vov, V.A.; Soderberg, O.; Hannula, S.-P. Stabilizing internal stress as the thermodynamic factor of martensite aging effects. Acta Mater. 2011, 59, 3593-3601.

37. L'vov, V.A.; Kosogor, A.; Barandiaran, J.M.; Chernenko, V.A. Destabilization of Ni-Mn-Ga martensite: Experiment and theory. Acta Mater. 2012, 60, 1587-1593. 
38. Liakos, J.K.; Saunders, G.A. Application of the Landau theory to elastic phase transitions. Philos. Mag. A 1982, 46, 217-242.

39. Gomonaj, E.V.; L'vov, V.A. Martensitic phase transition with two-component order parameter in a stressed cubic crystal. Phase Trans. 1994, 47, 9-21.

40. Barsch, G.R.; Krumhansl, J.A. Twin boundaries in ferroelastic media without interface dislocations. Phys. Rev. Lett. 1984, 53, 1069-1072.

41. Danilevich, A.G.; L'vov, V.A. Strong influence of ferromagnetic ordering and internal pressure on the elastic modulus of shape memory alloy. JMMM 2013, 333, 108-113.

42. Patel, J.R.; Cohen, M. Criterion for the action of applied stress in the martensitic transformation. Acta Metall. 1953, 1, 531-538.

43. Rohde, R.W.; Graham, R.A. The effect of hydrostatic pressure on the martensitic reversal of an iron-nickel-carbon alloy. Trans. Met. Soc. AIME 1969, 245, 2441-2445.

44. Otsuka, K.; Ren, X. Mechanism of martensite aging effects and new aspects. Mater. Sci. Eng. A 2001, 312, 207-218.

45. Pelegrina, J.L.; Ahlers, M. Influence of a constant stress during isothermal $\beta$ phase ageing on the martensitic transformation in a $\mathrm{Cu}-\mathrm{Zn}-\mathrm{Al}$ shape memory alloy. Scr. Mater. 2004, 50, 423-427.

46. Cahn, R.W. Metallic rubber bounces back. Nature 1995, 374, 120-121.

47. Picornell, C.; Pons, J.; Cesari, E. Effect of aging under compressive stress along [100] in Co-Ni-Ga single crystals. Funct. Mater. Lett. 2009, 2, 83-86.

48. Otsuka, K.; Ren, X. Physical metallurgy of Ti-Ni based shape memory alloys. Prog. Mater. Sci. 2005, 50, 511-678.

49. L'vov, V.A.; Glavatska, N.; Aaltio, I.; Söderberg, O.; Glavatskyy, I.; Hannula, S.-P. The role of anisotropic thermal expansion of shape memory alloys in their functional properties. Acta Mater. 2009, 57, 5605-5612.

50. Brill, T.M.; Mittelbach, S.; Assmus, W.; Mullner, M.; Luthi, B. Elastic properties of NiTi. J. Phys. Condens. Matter. 1991, 3, 9621-9627.

51. Chernenko, V.A.; Homenko, D.V.; L'vov, V.A.; Barandiaran, J.M. Specific heat of shape memory alloys with soft elastic moduli. J. Appl. Phys. 2011, 109, 013526:1-013526:6.

52. Lapshin, V.P.; Grishkov, V.N.; Lotkov, A.I. On certain anharmonic characteristics of B2-Phase Ti(Ni, Fe) alloys under hydrostatic pressure. Russ. Phys. J. 2000, 43, 999-1002.

53. Ren, X.; Otsuka, K. Origin of rubber-like behaviour in metal alloys. Nature 1997, 389, 579-582.

54. Ishibashi, H.; Kogachi, M.; Ohba, T.; Ren, X.; Otsuka, K. Vacancy migration and long-range ordering due to ageing in AuCd shape memory alloys. Mater. Sci. Eng. A 2002, 329-331, $568-572$.

55. Tadaki, K.; Otsuka, K.; Shimizu, K. Shape memory alloys. Ann. Rev. Mater. Sci. 1988, 18, 25-45.

56. Otsuka, K.; Ren, X. Mechanism of martensite aging effect. Scr. Mater. 2004, 50, 207-212.

57. Murakami, Y.; Nakajima, Y.; Otsuka, K.; Ohba, T.; Matsuo, R.; Ohshima, K. Characteristics and mechanism of martensite ageing effect in Au-Cd alloys. Mater. Sci. Eng. A 1997, 237, 87-101.

58. Ohta, T. Theory of rubber-like elasticity in shape memory alloys. Mater. Sci. Eng. A 2001, 312, $57-65$. 
59. Ren, X.; Otsuka, K. Universal symmetry property of point defects in crystals. Phys. Rev. Lett. 2000, 85, 1016-1019.

60. Xue, D.; Zhou, Y.; Ding, X.; Otsuka, K.; Sun, J.; Ren, X. Martensite aging effects on the dynamic properties of $\mathrm{Au}-\mathrm{Cd}$ shape memory alloys: Characteristics and modelling. Acta Mater. 2011, 59, 4999-5011.

61. Xue, D.; Zhou, Y.; Ding, X.; Lookman, T.; Sun, J.; Ren, X. Aging and deaging effects in shape memory alloys. Phys. Rev. B 2012, 86, 184109:1-184109:11.

62. Kosogor, A.; Xue, D.; Zhou, Y.; Ding, X.; Otsuka, K.; L’vov, V.A.; Sun, J.; Ren, X. Impact of the volume change on the aging effects in $\mathrm{Cu}-\mathrm{Al}-\mathrm{Ni}$ martensite: experiment and theory. $J$. Phys. Condens. Matter, submitted for publication, 2013.

63. Otsuka, K.; Ren, X.; Murakami, Y.; Kawano, T.; Ishii, T.; Ohba, T. Composition dependence of the rubber-like behavior in $\zeta_{2}^{\prime}$-martensite of AuCd alloys. Mater. Sci. Eng. A 1999, 273, $558-563$.

64. Ren, X.; Otsuka, K. The role of softening in elastic constant c44 in martensitic transformation. Scr. Mater. 1998, 38, 1669-1675.

65. Otsuka, K.; Ren, X. Martensitic transformations in nonferrous shape memory alloys. Mater. Sci. Eng. A 1999, 273-275, 89-105.

66. Otsuka, K.; Shimizu, K. Morphology and crystallography of thermoelastic Cu-Al-Ni martensite analyzed by the phenomenological theory. Mat. Trans. JIM 1974, 15, 103-108.

67. Van Humbeeck, J.; Janssen, J.; Mwamba, N.; Delaey, L. The stabilisation of step-quenched copper-zinc-aluminum martensite part I: The reverse transformation temperatures. Scr. Metall. 1984, 18, 893-898.

68. Nakata, Y.; Yamamoto, O.; Shimizu, K. Effect of aging in Cu-Zn-Al shape memory alloys. Mat. Trans. JIM 1993, 34, 429-437.

69. Kokorin, V.V.; Kozlova, L.E.; Titenko, A.N. Temperature hysteresis of martensite transformation in aging $\mathrm{Cu}-\mathrm{Mn}-\mathrm{Al}$ alloy. Scr. Mater. 2002, 47, 499-502.

70. Kustov, S.; Pons, J.; Cesari, E.; Van Humbeeck, J. Pinning-induced stabilization of martensite: Part I. Stabilization due to static pinning of interfaces. Acta Mater. 2004, 52, 3075-3081.

71. Chernenko, V.A.; Pons, J.; Cesari, E.; Zasimchuk, I.K. Transformation behaviour and martensite stabilization in the ferromagnetic Co-Ni-Ga Heusler alloy. Scr. Mater. 2004, 50, 225-229.

72. Chernenko, V.A.; Villa, E.; Besseghini, S.; Barandiaran, J.M. Giant two-way shape memory effect in high-temperature Ni-Mn-Ga single crystal. Phys. Proced. 2010, 10, 94-98.

73. Hsieh, S.F.; Chang, W.K. Martensitic transformation of an aged/thermal-cycled Ti30.5Ni49.5Zr10Hf10 Shape Memory Alloy. J. Mater. Sci. 2007, 37, 2851-2856.

74. Wayman, C.M.; Cornelis, I.; Shimizu, K. Transformation behavior and the shape memory effect in thermally cycled TiNi. Scr. Metall. 1972, 6, 115-122.

75. Miyazaki, S.; Igo, Y.; Otsuka, K. Effect of thermal cycling on the transformation temperatures of Ti-Ni alloys. Acta Metall. 1986, 34, 2045-2051.

76. Hsieh, S.F.; Wu, S.K.; Lin, H.C. Martensitic transformation of a Ti-rich $\mathrm{Ti}_{51} \mathrm{Ni}_{47} \mathrm{Si}_{2}$ shape memory alloy. J. Alloys Compd. 2002, 335, 254-261.

77. Perkins, J.; Muesing, W.E. Martensitic transformation cycling effects in $\mathrm{Cu}-\mathrm{Zn}-\mathrm{Al}$ shape memory alloys. Met. Trans. A 1983, 14, 33-36. 
78. Chernenko, V.A.; Kokorin, V.V.; Babii, O.M.; Zasimchuk, I.K.; Phase diagrams in the $\mathrm{Ni}-\mathrm{Mn}-\mathrm{Ga}$ system under compression. Intermetallics 1998, 6, 29-34.

79. Landau, L.D.; Lifshitz, E.M. Course of Theoretical Physics; Volume 5 Statistical Physics; Pergamon Press: New York, NY, USA, 1980.

80. L'vov, V.A.; Matsishin, N.; Glavatska, N. Thermoelastic behaviour of martensitic alloy in the vicinity of critical point in the stress-temperature phase diagram. Phase Trans. 2010, 83, 293-301.

81. Kosogor, A.; Matsishin, N.J.; L'vov, V.A. Modelling of hysteresis loops taken during the stress- and temperature-induced martensitic transformations. Phase Trans. 2013, in press.

82. L'vov, V.A.; Gomonaj, E.V.; Chernenko, V.A. A phenomenological model of ferromagnetic martensite. J. Phys. Condens. Mat. 1998, 10, 4587-4596.

83. Chernenko, V.A.; L'vov, V.A.; Pasquale, M.; Besseghini, S.; Sasso, C.; Polenur, D.A. Magnetoelastic behavior of Ni-Mn-Ga martensitic alloys. Int. J. Appl. Electromagn. Mech. 2000, $12,3-8$.

84. Chernenko, V.A.; L'vov, V.A.; Zagorodnyuk, S.P.; Takagi, T. Ferromagnetism of thermoelastic martensites: Theory and experiment. Phys. Rev. B 2003, 67, 064407:1-064407:6.

85. Buchelnikov, V.D.; Khovailo, V.V.; Takagi, T. The thermal expansion coefficient and volume magnetostriction of Heusler Ni2MnGa alloys. JMMM 2006, 300, e459-e461.

86. Chernenko, V.A.; Amengual, A.; Cesari, E.; Kokorin, V.V.; Zasimchuk, I.K. Thermal and magnetic properties of stress-induced martensites in Ni2MnGa alloys. J. Phys. IV 1995, 5, 95-98.

87. Fukuda, T.; Maeda, H.; Yasui, M.; Kakeshita, T. Influence of magnetocrystalline anisotropy on martensitic transformation under magnetic field of single-crystalline Ni2MnGa. Scr. Mater. 2009, 60, 261-263.

88. Seguí, C.; Chernenko, V.A.; Pons, J.; Cesari, E. Low-Temperature-Induced intermartensitic phase transformations in Ni-Mn-Ga single crystal. JMMM 2005, 290, 811-815.

89. Seguí, C.; Chernenko, V.A.; Pons, J.; Cesari, E. Two-Step martensitic transformation in Ni-Mn-Ga alloys. J. Phys. IV 2003, 112, 903-906.

90. Chernenko, V.A.; Cesari, E.; Pons, J.; Seguí, C. Phase transformations in rapidly quenched Ni-Mn-Ga alloys. Mat. J. Res. 2000, 15, 1496-1504.

91. Chernenko, V.A.; Cesari, E.; Kokorin, V.V.; Vitenko, I.N. The development of new ferromagnetic shape memory alloys in Ni-Mn-Ga system. Scr. Met. Mat. 1995, 33, 1239-1244.

92. Cesari, E.; Salas, D.; Kustov, S. Entropy changes in ferromagnetic shape memory alloys. Mat. Sci. Forum 2011, 684, 49-60.

93. Recarte, V.; Pérez-Landazábal, J.I.; Gómez-Polo, C.; Sánchez-Alarcos, V.; Cesari, E.; Pons, J. Vibrational and magnetic contributions to the entropy change associated with the martensitic transformation of $\mathrm{Ni}-\mathrm{Fe}-\mathrm{Ga}$ ferromagnetic shape memory alloys. J. Phys. Condens. Matter 2010, 22, 416001:1-416001:7.

94. Liu, Z.H.; Hu, H.N.; Liu, G.D.; Cui, Y.T.; Zhang, M.; Chen, J.L.; Wu, G.H. Electronic structure and ferromagnetism in the martensitic-transformation material Ni2FeGa. Phys. Rev. B 2004, 69, 134415. 
95. Seguí, C.; Cesari, E. Composition and atomic order effects on the structural and magnetic transformations in ferromagnetic Ni-Co-Mn-Ga shape memory alloys. J. Appl. Phys. 2012, 111, 043914:1-043914:7.

96. Barandiaran, J.M.; Chernenko, V.A.; Cesari, E.; Salas, D.; Lazpita, P.; Gutierrez, J.; Orue, I. Magnetic influence on the martensitic transformation entropy in Ni-Mn-In metamagnetic alloy. Appl. Phys. Lett. 2013, 102, 071904:1-071904:4.

97. Gruner, M.E.; Entel, P. Impact of local lattice distortions on the structural stability of Fe-Pd magnetic shape-memory alloys. Phys. Rev. B 2011, 83, 214415:1-214415:11.

(C) 2013 by the authors; licensee MDPI, Basel, Switzerland. This article is an open access article distributed under the terms and conditions of the Creative Commons Attribution license (http://creativecommons.org/licenses/by/3.0/). 